\title{
The Novel Phosphate and Bile Acid Sequestrant Polymer SAR442357 Delays Disease Progression in a Rat Model of Diabetic Nephropathy ${ }^{\text {[ }}$
}

\author{
Tamara R. Castañeda, María Méndez, lan Davison, Ralf Elvert, Uwe Schwahn, \\ Galina Boldina, Corinne Rocher, Petra Scherer, Kuldeep Singh, ${ }^{1}$ Dinesh S. Bangari, \\ Mechthilde Falkenhahn, Aimo Kannt, ${ }^{2}$ Anish Konkar, ${ }^{3}$ Philip J. Larsen, ${ }^{4}$ Cynthia Arbeeny, \\ Pradeep K. Dhal, and (1)Thomas Hübschle
}

R\&D Diabetes (T.R.C., R.E., A.Ka., A.Ko., P.J.L., C.A., T.H.), Integrated Drug Discovery (M.M.), Biomarkers and Clinical Bioanalysis (U.S.), Translational In Vivo Models, Global Discovery Pathology (P.S.), and Global Research Project Management (M.F.), Sanofi-Aventis Deutschland GmbH, Industriepark Höchst, Frankfurt, Germany; C\&BD Haverhill Operations, Sanofi GB Genzyme Limited, Haverhill, Suffolk, United Kingdom (I.D.); R\&D Translational Sciences France, Bioinformatics, Sanofi, Chilly-Mazarin Cedex, France (C.R.); Translational In Vivo Models, Global Discovery Pathology, Framingham, Massachusetts (K.S., D.S.B.); and Pharmaceutical Development Platform, Sanofi Global R\&D, Waltham, Massachusetts (P.K.D.)

Received August 14, 2020; accepted November 10, 2020

\section{ABSTRACT}

As a gut-restricted, nonabsorbed therapy, polymeric bile acid sequestrants (BAS) play an important role in managing hyperlipidemia and hyperglycemia. Similarly, nonabsorbable sequestrants of dietary phosphate have been used for the management of hyperphosphatemia in end-stage renal disease. To evaluate the potential utility of such polymer sequestrants to treat type 2 diabetes (T2D) and its associated renal and cardiovascular complications, we synthesized a novel polymeric sequestrant, SAR442357, possessing optimized bile acid (BA) and phosphate sequestration characteristics. Long-term treatment of T2D obese ${ }^{\mathrm{C}}$ Zucker fatty/ Spontaneously hypertensive heart failure F1 hybrid (ZSF1) with SAR442357 resulted in enhanced sequestration of BAs and phosphate in the gut, improved glycemic control, lowering of serum cholesterol, and attenuation of diabetic kidney disease (DKD) progression. In comparison, colesevelam, a BAS with poor phosphate binding properties, did not prevent DKD progression, whereas losartan, an angiotensin II receptor blocker that is widely used to treat DKD, showed no effect on hyperglycemia. Analysis of hepatic gene expression levels of the animals treated with SAR442357 revealed upregulation of genes responsible for the biosynthesis of cholesterol and BAs, providing clear evidence of target engagement and mode of action of the new sequestrant. Additional hepatic gene expression pathway changes were indicative of an interruption of the enterohepatic BA cycle. Histopathological analysis of ZSF1 rat kidneys treated with SAR442357 further supported its nephroprotective properties. Collectively, these findings reveal the pharmacological benefit of simultaneous sequestration of BAs and phosphate in treating T2D and its associated comorbidities and cardiovascular complications.

\section{SIGNIFICANCE STATEMENT}

A new nonabsorbed polymeric sequestrant with optimum phosphate and bile salt sequestration properties was developed as a treatment option for DKD. The new polymeric sequestrant offered combined pharmacological benefits including glucose regulation, lipid lowering, and attenuation of DKD progression in a single therapeutic agent.
This work received no external funding.

All authors are current or former employees of Sanofi. No author has an actual or perceived conflict of interest with the contents of this article.

${ }^{1}$ Current affiliation: WuXi AppTec Inc., Cambridge, Massachusetts.

${ }^{2}$ Current affiliation: Fraunhofer IME, Translational Medicine and Pharmacology, Frankfurt, Germany.

${ }^{3}$ Current affiliation: Eli Lilly and Company, Indianapolis, Indiana.

${ }^{4}$ Current affiliation: Bayer AG, Pharmaceutical R\&D, Wuppertal, Germany. https://doi.org/10.1124/jpet.120.000285.

S This article has supplemental material available at jpet.aspetjournals.org.

\section{Introduction}

Increased prevalence of diabetes has resulted in increased incidence of renal impairments leading to diabetic kidney disease (DKD). As a microvascular complication, DKD often progresses to end-stage renal disease (ESRD), requiring dialysis or kidney replacement therapy. DKD has become the leading cause of ESRD (Cameron, 2006, USRDS, 2015; Alicic et al., 2017), with approximately $35 \%$ of all deaths from chronic kidney disease (CKD) being attributed to DKD (Thomas, 2019). Moreover, a higher rate of cardiovascular (CV) mortality is observed among patients with DKD with

ABBREVIATIONS: BA, bile acid; BAS, bile acid sequestrant; BES, N,N-Bis(2-hydroxyethyl)-2-aminoethanesulfonic acid; CKD, chronic kidney disease; CV, cardiovascular; DKD, diabetic kidney disease; ESRD, end-stage renal disease; FDR, false discovery rate; GC, glycocholic acid; GCDC, glycochenodeoxycholic acid; Gl, gastrointestinal; HbA1c, Glycated Haemoglobin; KIM 1, Kidney Injury Molecule 1; PAA, poly(allylamine); PAS, periodic acid-Schiff; PDA, poly(diallylamine); T2D, type 2 diabetes; ZSF1, Zucker fatty/Spontaneously hypertensive heart failure F1 hybrid. 
ESRD compared with non-DKD ESRD (Giorda et al., 2018; Thomas, 2019). With nearly 160 million patients with DKD worldwide, it is projected there will be 212 million by 2040 (Alicic et al., 2017).

Currently, there is no cure for CKD, including DKD. The available treatment options are limited to relieving the symptoms and slowing down disease progression. These include management of hyperglycemia and hypertension and use of renin-angiotensin-aldosterone system inhibitors and sodium-dependent glucose cotransporter-2 inhibitors (Lytvyn et al., 2020). Losartan, an angiotensin II type 1 receptor blocker, is one of the first-line therapies employed to ameliorate hypertension-associated CV disease and DKD. By inhibiting arteriolar contraction and sodium retention, losartan decreases proteinuria, albuminuria, and markers of tubular damage (Nielsen et al., 2011; Katsiki et al., 2018, Francischetti et al., 2020). Moreover, diuretics, cholesterollowering drugs, and phosphate binders are used to treat CKD (Holman et al., 2008; Bilous, 2008; Floege, 2016; Alicic et al., 2017).

Hyperphosphatemia is a manifestation of renal failure and positively correlates with progression to ESRD. In ESRD, hyperphosphatemia is associated with secondary hyperparathyroidism, metabolic bone disease, and progressive vascular calcification, resulting in significant CV morbidity and mortality (Block et al., 1998, 2004; Goodman et al., 2000; Hruska et al., 2008). Therefore, pharmacological intervention to restrict phosphate absorption using phosphate binders is a mainstay of ESRD treatment. Sevelamer is a nonabsorbable polymeric phosphate binder used to lower serum phosphorus levels in patients with CKD on dialysis. Sevelamer also exhibits pleiotropic beneficial effects. For example, sevelamer improves lipid and glucose metabolism (Vlassara et al., 2012; Brønden et al., 2018), reduces inflammation and oxidative stress (Caglar et al., 2008; Sun et al., 2009; Navarro-González et al., 2011; Vlassara et al., 2012; Yilmaz et al., 2012; Rastogi, 2013; Brønden et al., 2020), clears uremic toxins (Garg et al., 2005; Vlassara et al., 2012), and prevents progression of aortic and coronary calcification (Chertow et al., 2002; Moe and Chen, 2004; Floege and Ketteler, 2004; Asmus et al., 2005; Rastogi, 2013). These benefits result in significant survival in sevelamer-treated patients compared with those receiving metal-based phosphate binders (Block et al., 2007; Di Iorio et al., 2012, 2013; Rodríguez-Osorio et al., 2015; Floege, 2016; Patel et al., 2016).

Colesevelam is a nonabsorbed polymeric cholesterollowering drug, which belongs to the class of bile acid sequestrants (BAS). Colesevelam is approved for the treatment of hyperlipidemia and T2D. Its therapeutic action is derived from its ability to interrupt enterohepatic circulation of BAs by sequestering and removing BAs from the gastrointestinal (GI) tract (Rosenbaum et al.1997; Stroeve et al., 2010; Herrema et al., 2010; Potthoff et al., 2013). Colesevelam improves glycemia by eliciting changes in BAS-mediated modulation of nuclear farnesoid $\mathrm{X}$ bile acid receptordependent signaling pathways that regulate hepatic gluconeogenesis (Takebayashi et al., 2010; Handelsman, 2011; Kodera et al., 2011; Hansen et al., 2014; Inzucchi et al., 2015). Moreover, colesevelam enhances the conversion of intestinal BA toward secondary BAs, thereby stimulating secretion of the incretin, glucagon-like peptide 1 (GLP-1) from enteroendocrine L cells (Fuchs et al., 2018).
Colesevelam and sevelamer possess similar chemical attributes and thus bind similar substrates in the gut; however, withdifferent binding affinities and capacities towards these substrates. Therefore, we hypothesized that the discovery of a novel polymeric sequestrant that combines the efficacy and pleiotropic benefits of phosphate and BA binding with optimum capacity and affinity may offer significant clinical benefit in delaying progression to ESRD and reducing CV risk. We have synthesized a novel polymeric sequestrant (SAR442357) with optimum physicochemical properties as well as optimum binding affinity and capacity toward both phosphate and BA anions. Such a compound could serve as an innovative new therapy for the treatment of DKD with potentially fewer side effects. Here, we report the preclinical results and mechanistic insights into the pharmacological action of SAR442357 as a treatment of DKD using the ZSF1 rat model, which manifests disease progression and pathology of human DKD, including hypertension, obesity, hyperglycemia, and T2D mellitus (Bilan et al., 2011; van Dijk et al., 2016; Su et al., 2016; Dower et al., 2017).

\section{Material and Methods}

\section{Synthesis of Hydrogels}

Poly(allylamine hydrochloride) and poly(diallylamine hydrochloride) were synthesized by free radical polymerization of allylamine and diallylamine, respectively, in the forms of their hydrochloride salts by following reported procedures (Harada and Hasegawa, 1984; Jang and Rasmussen, 1998). The crosslinked polymer networks made up of PAA and PDA components are described here using SAR442357 as the specific example. Detailed parameters for the syntheses of a series of such hydrogels are summarized in Supplemental Table 1. In a jacketed vessel with lid, a mixture of poly(allylamine hydrochloride) and poly(diallylamine hydrochloride) (14.3:1 molar ratio) was diluted with distilled water to achieve the desired polymer concentration of $40 \%(\mathrm{w} / \mathrm{v})$. The reaction mixture was subsequently warmed to $30^{\circ} \mathrm{C}$, and the $\mathrm{pH}$ of the solution was adjusted to 11.0 by adding sodium hydroxide $(\mathrm{NaOH})$ pellets [for smaller batches, $50 \%$ (w/v) aqueous $\mathrm{NaOH}$ solution can be used] while maintaining the temperature of the reaction mixture at $25^{\circ} \mathrm{C}$. The temperature of the stirred reaction mixture was slowly brought to room temperature, followed by the addition of an appropriate amount of epichlorohydrin $(14.3 \mathrm{mmol})$ to the reaction mixture via a syringe. The temperature of the reaction mixture was subsequently brought to $40^{\circ} \mathrm{C}$ and stirred until the reaction mixture became a gel. At this point, the stirring was stopped, and reaction mixture was cooled down to room temperature. The gel was placed in a glass drying dish and allowed to cure overnight at $20^{\circ} \mathrm{C}$ in a vacuum oven. The gel was subsequently broken into smaller pieces and pushed through a $200-\mu \mathrm{m}$ sieve to produce smaller particles. The polymer particles were suspended in deionized water, and the $\mathrm{pH}$ of the suspension was adjusted to 13.0 by the addition of $50 \%(\mathrm{w} / \mathrm{v})$ aqueous $\mathrm{NaOH}$ solution. The resulting suspension was stirred for 20 minutes and filtered over filter paper (113 grade). The gel particles were subjected to a series of washing (using deionized water) and filtration steps until the conductivity of the suspension reached a value of $<50 \mu \mathrm{S} / \mathrm{cm}$. Subsequently, the suspension was bubbled with appropriate amounts of $\mathrm{CO}_{2}$ gas to reach the carbonation level of $\sim 13 \%$. The gel particles were filtered and dried at $60^{\circ} \mathrm{C}$ under reduced pressure to constant weight. The dried particles were ground using a mill grinder or cryomilling and were fractioned into particles of different sizes using sieves of appropriate mesh size $(50-100 \mu \mathrm{m})$.

\section{Characterization of Hydrogels}

Elemental analyses were carried out at Quantitative Technologies Inc (QTI) laboratories (Whitehouse, NJ). The percentage of carbonate counter ions was determined by thermogravimetric analysis. Particle size 
measurement was carried out using Malvern Mastersizer 3000 instrument (Malvern Panalytical Ltd., Malvern, UK). Solid-state magic angle spinning NMR spectra of the swollen gels (in deuterium oxide (D2O)) were measured using a Bruker AVANCE $400 \mathrm{MHz}$ NMR spectrometer (Bruker BioSpin GmbH, Ettlingen, Germany). Equilibrium swelling indices of these hydrogels in PBS medium were determined by following a published method (Polomoscanik et al., 2012). Total titratable amine contents in the hydrogels were determined by potentiometric titration. The titration process involves conversion of the polymer gel into its $\mathrm{HCl}$ salt by treatment with excess $\mathrm{HCl}$. The resulting $\mathrm{HCl}$ salt of the gel is titrated with $1.0 \mathrm{~N} \mathrm{NaOH}$. Two inflection points were obtained on the titration curve, the first one corresponding to $\mathrm{HCl}$ (strong acid) neutralization and the second one corresponding to amine hydrochloride (weak acid) neutralization. The amount of amine groups in the polymer gel is estimated as a difference between the second and the first inflection points.

\section{Equilibrium Binding of Phosphate and Bile Acid Salts}

Equilibrium binding capacities of the hydrogels toward bile salts such as sodium salts of glycocholic acid (GC) and glycochenodeoxycholic acid (GCDC) were determined according to a published procedure (Braunlin et al., 2002). Equilibrium binding capacities of the hydrogels for phosphate ions were measured in N,N-Bis(2-hydroxyethyl)-2-aminoethanesulfonic acid ( ${ }^{\mathrm{b}} \mathrm{BES}$ ) buffer using potassium phosphate monobasic $\left(\mathrm{KH}_{2} \mathrm{PO}_{4}\right)$ as the substrate, followed by estimation of unbound phosphate ions by ion chromatography. The experimental details for these measurements are described elsewhere (Swearingen et al., 2004).

\section{Animals}

Eight-week-old male Zucker fatty/Spontaneously hypertensive heart failure F1 hybrid (ZSF1) rats (Charles River, Kingston, NY) were housed under a 12-hour light/dark cycle (lights on at $6 \mathrm{AM}$ ) with ad libitum access to a standard diet for this animal model (modified PMI 5008 diet; Ssniff, Soest, Germany) and tap water. Body weight, tail blood, urine, and fecal basal values were collected at 10 to 11 weeks of age. Subsequently, the obese ZSF1 rats were randomized to four groups based on body weight, random blood glucose, ${ }^{\mathrm{a}} \mathrm{HbA1c}(\%)$, and urinary albumin. From 12 weeks of age, the corresponding groups were treated daily with losartan (by mouth, $10 \mathrm{mg} / \mathrm{kg}$ in the diet) (Hennig, Germany), colesevelam (2\% in the diet), or SAR442357 (2\% or $4 \%$ in the diet) for 3 months. The drugs in the form of dry powder were dry-mixed with the powdered standard Sniff diet at appropriate ratios. After homogenization in dry form, the drug-containing diets as well as drug-free diets were cold-pelleted. Selection of the doses used for the polymers was based on published literature on polymeric sequestrants (such as colesevelam, colestilan, and also sevelamer) that, for example, described the $2 \%$ colesevelam dose as an efficacious dose in rodent models when added to the diet of the animals (Herrema et al., 2010; Meissner et al., 2011). Initially, each group had 10 animals. Based on urine albumin/creatinine values, two nonprogressor rats were removed from the obese ZSF1 control group. Measurements were taken at baseline (age 11 weeks) and at the end of second and third month of treatment (age 20 and 24 weeks, respectively). All experiments were approved by the Ethics Committee of the State Ministry of Agriculture, Nutrition and Forestry (State of Hesse, Germany).

\section{Body Weight, Blood, and Serum Parameters}

Body weight was monitored weekly with a balance (Mettler Toledo, Modell New Classic MF, Greifensee, Switzerland). Blood glucose was measured with a glucometer (ACCU-CHEK Aviva, model: NC; Roche Diagnostics, Mannheim, Germany) and HbA1c (\%) with a Cobas 6000 analyzer (Roche Diagnostics). Final measurements were performed before the animals were anesthetized.
Tail blood collection was performed for basal and 20-week time points. For final measurements, rats were euthanized in an ad libitum state under deep isoflurane anesthesia by aortic exsanguination. Blood was collected (S-Monovette Z-Gel, clot activator; Sarstedt, Nümbrecht, Germany), coagulated for 20 minutes at room temperature, and centrifuged $\left(4^{\circ} \mathrm{C}, 10\right.$ minutes, $\left.4000 \mathrm{rpm}\right)$ for serum collection. Serum triglycerides, total cholesterol, inorganic phosphate, urea, and creatinine were measured (Cobas 6000 analyzer; Roche Diagnostics). Measurements of serum triglycerides and total cholesterol at the 2month time point for the obese ZSF1 SAR442357-treated (2\%) group and 3-month time points for the obese ZSF1 colesevelam-treated (2\%) group were not recorded because of the insufficient sample volume. One measurement for serum inorganic phosphate was missed at the 3month time point for the obese ZSF1 colesevelam (2\%) group and at the 2-month time point for the lean ZSF1 and obese ZSF1 (2\% and 4\%) groups because of the lack of adequate sample volume.

Serum insulin was measured with an ELISA kit (Mercodia rat/mouse insulin Fluorescent Immunoassay (FIA) number 10-1248-10) following the manufacturer's guidelines. For insulin measurements, one outlier value was removed from the obese ZSF1 SAR442357 (2\%) group from the 2-month time point.

\section{Gene Expression Analysis}

Sequencing of mRNA. Liver samples were collected in RNAlater solution (Invitrogen, Thermo Fisher Scientific, Darmstadt, Germany) and stored at $-80^{\circ} \mathrm{C}$ until further processing. Total RNA was extracted from the samples using RNeasy Mini Kit (QIAGEN Inc., Hilden, Germany) according to the manufacturer's instructions. Total RNA purity and concentration was determined on a Nanodrop8000 spectrophotometer (Thermo Fisher Scientific) with subsequent RNA quality control with an Agilent 2100 bioanalyzer using an Agilent RNA 6000 Nano Kit (Agilent technologies, Santa Clara, CA). RNAs with a RNA integrity number (RIN) score of 8 or higher were used for RNA sequencing. Library preparation was done with the Illumina TruSeq RNA Sample Prep Kit v2, and subsequent sequencing was done on an Illumina NextSEq 550 (Illumina, Inc., San Diego, CA).

Ribonucleic acid sequence (RNA-Seq) data analysis was performed using ArrayStudio (QIAGEN Inc.). Briefly, raw data quality control is performed, followed by a filtering step to remove reads corresponding to ribosomal ribonucleic acids (rRNAs) as well as reads that have a low quality score or are shorter than 25 nucleotides. Reads were further mapped to the rat genome (rn6) using Omicsoft Sequence Aligner version 4 (OSA4). (Hu et al., 2012) (Omicsoft Sequence Aligner, version 4) and quantified using ENSEMBL gene model of transcriptome, with parameters enabling identification of new splice variants. Differential analysis of gene expression was performed at the gene level using Voom module integrated to Array Studio (Law et al., 2014). This module uses Voom in the Limma R/Bioconductor package.

\section{Bomb Calorimetry}

Combustion of Food and Feces. Food consumption was measured and corrected by separating the unconsumed food from feces. Because of the technical issues during monitoring, one measurement for food consumption was excluded from the obese ZSF1 colesevelam (2\%) group at 2-and 3-month time points as well as five measurements from the obese ZSF1 losartan $(10 \mathrm{mg} / \mathrm{kg}$ ) group. Samples of chow and collected feces $(\sim 1 \mathrm{~g})$ were dried at $60^{\circ} \mathrm{C}$ for up to 7 days, homogenized in a coffee grinder, and squeezed to pills. Energy contents in these samples were determined in an oxygen bomb calorimeter (model 6300; Parr Instruments Deutschland GmbH, Frankfurt a.M., Germany). For the analysis of solid feces samples, the bomb calorimeter was calibrated using benzoic acid for calorimetrical determination with a guaranteed caloric value of $26.47 \mathrm{~kJ} \mathrm{~g}^{-1}$ (Sigma-Aldrich, Darmstadt, Germany). Energy uptake (Eup) was determined as the product of food consumed and the caloric value of the food. The energy loss was 
defined as the sum of fecal (Eloss fec) and urinary caloric loss (Eloss urine, for details see below) and calculated from the feces/urine produced per 24 hours multiplied by its respective energy content. To obtain energy metabolized (Emet), the energy content of feces and urine was subtracted from energy intake, calculated as Emet $=$ Eup - (Eloss fec + Eloss urine) (Drozdz et al., 1975). The utilization of food energy is expressed as food efficiency corresponding to the percentage of metabolized energy from energy intake.

Combustion of Urine. Urinary samples were collected using metabolic chambers to separate urine from food and feces. For determination of urinary caloric value, samples were usually freezedried, pulverized, and combusted in bomb calorimeters to obtain an estimate of excretory energy losses (Raman et al., 2007; Singh et al., 2009). Because of the low urinary volume of the lean rats, we developed a procedure using cotton coils as a combustion aid (Heiland Vet GmbH, Germany). Depending on sample volume available, two different sizes of coils were used: $8 \mathrm{~mm}$ diameter $\times 19 \mathrm{~mm}$ length (for low volumes) and $10 \mathrm{~mm} \times 19 \mathrm{~mm}$ (for high volumes). The caloric value of the combustion aid was $16.49 \mathrm{~kJ} \mathrm{~g}^{-1}$. Results of urinary samples were corrected automatically for the value of the aid. Calibration and testing for validity were performed as previously reported (Elvert et al., 2013). The analysis of urine followed the same procedure used for the feces samples. One outlier was excluded from the obese ZSF1 colesevelam (2\%) group at the 2-month time point.

\section{Analysis of Fecal Bile Acids, Fecal Phosphate, and Fecal Fatty Acids}

Fecal samples were collected over 24 hours from single-housed rats using metabolic cages (model 3701M001; Techniplast, Buguggiate VA, Italy). The feces were dried, weighed, and frozen at $-20^{\circ} \mathrm{C}$ for later analysis. Extraction and measurement of fecal total bile acids were performed according to the method described by Dvir et al. (2000) with minor modifications. Fecal inorganic phosphate and fecal volatile fatty acids were measured from the identical extraction solutions.

Briefly, dried feces were homogenized in a potassium hydroxide solution ( $\mathrm{pH}$ 11.0) with an Ultra-Turrax disperser (model UltraTurrax T25 basic; IKA-Works Inc., Wilmington, NC) to obtain a uniform slurry with a target concentration of $50 \mathrm{mg}$ feces per milliliter of homogenate. Bile acid, phosphate, and free fatty acid extraction was performed by agitating the slurry on a shaker using the solvents dichloromethane and methanol. An aliquot of the feces homogenate containing $500 \mathrm{mg}$ feces was extracted over 4 hours at room temperature with $5 \mathrm{ml}$ of a dichloromethane:methanol $(2: 1, \mathrm{v} / \mathrm{v})$ mixture. In total, $2 \mathrm{ml}$ of aqueous potassium chloride solution $(3.7 \mathrm{~g} / \mathrm{l})$ was added, and samples were incubated further for 1 hour and then centrifuged at $1500 \mathrm{~g}$ for 10 minutes. The aqueous supernatant phase was removed, and its volume was determined and then stored at $-20^{\circ}$ C. Finally, samples were thawed at $40^{\circ} \mathrm{C}$, centrifuged at $1500 \mathrm{~g}$ for 10 minutes, and then analyzed for total bile acid, inorganic phosphate, and total nonesterified fatty acid concentrations using commercially available enzymatic and colorimetric assay kits on a chemistry analyzer (Beckman Coulter AU680; Beckman Coulter Inc., Clare, Ireland).

\section{Urine Parameters}

Urine was collected in metabolic cages (model 3701M001; Techniplast) after 8 and 16 hours to have in total 24 hours of urine collection. Urine inorganic phosphate, glucose, total protein, albumin, and creatinine were measured using commercially available enzymatic assay kits and normalized by 24-hour urine volume (Beckman Coulter AU680; Beckman Coulter Inc.). Basal urine measurements were missed for one rat of the obese ZSF1 SAR442357 (2\%) group because of the accidental loss of the sample. Furthermore, one basal value was missing from the lean ZSF1 control group because albumin was under the lower limit of detection, and one outlier value for inorganic phosphate at the 2-month time point was removed from the obese ZSF1 losartan group.

Urine KIM1 $1^{\mathrm{d}}$ and cystatin $\mathrm{C}$ were measured with an ELISA kit (Quantikine ELISA, catalog number RKM100 for KIM1, catalog number MSCTC0 for cystatin C; R\&D Systems), following the manufacturer's guidelines. Basal urine measurements were missed for one rat from the obese ZSF1 SAR442357 (2\%) group because of the accidental loss of the sample and one KIM1 value of the obese ZSF1 losartan group at 3-month time point for being an outlier.

Both kidneys were removed after euthanasia and weighed (model $\times 204$; Mettler Toledo).

\section{Histology and Pathologic Analysis}

Kidneys were sampled and fixed in $4 \%$ neutral buffered formaldehyde for 48 hours and embedded in paraffin. Cross sections were obtained from each sample. Afterward, the dehydration of the tissue was done automatically with a vacuum infiltration processor (VIP) tissue processor (Sakura Tissue-Tek VIP 5 Tissue Processor; Sakura, Torrance, CA) for approximately 20 hours followed by the blocking procedure. Slice sections $4 \mu \mathrm{m}$ thick were taken from each sample and dried overnight on a stretching table at $45^{\circ} \mathrm{C}$.

Staining was performed automatically using a Gemini AS automatic slide stainer (Thermo Fisher Scientific, Runcorn, Great Britain).

Hematoxylin and Eosin Staining. Tissue deparaffination was carried with xylene three times for 3 minutes, followed by rehydration in decreasing ethanol solutions of $100 \%, 95 \%$, and $70 \%$ for 1 minute each. Staining of the nuclei was done with Haemalum from Mayer (blue) for 5 minutes, followed by rinsing with distilled water for 10 minutes and ascending ethanol solutions of $70 \%$ and $99 \%$. Final tissue staining was done with eosin (red) for 30 seconds, and ethanol and xylene were used three times for dehydration before permanent covering.

Periodic Acid-Schiff Staining. Tissue deparaffination was done with xylene three times for 3 minutes, followed by rehydration in decreasing ethanol solutions of $100 \%, 95 \%$, and $70 \%$ for 1 minute each. For preservation, the slices were exposed to periodic acid for 10 minutes and subsequently rinsed with tap water for 1 minute. Staining of glycoproteins was done with Schiff's reagent (pink) for 10 minutes, followed by rinsing with distilled water for 1 minute. Staining of the nuclei was done with Haemalum (blue) for 2 minutes, followed by rinsing with distilled water for 1 minute three times, ethanol and xylene for dehydration, and permanent covering.

For pathology analysis, whole-slide scanned $(20 \times)$ images were evaluated qualitatively and semiquantitatively by two board-certified veterinary pathologists (K.S. and D.S.B.). Criteria for semiquantitative evaluation included assessment based on the severity of a morphologic change and the extent of tissue involvement. For each rat, four parameters were assessed independently: glomerular pathology, tubular pathology, tubular protein casts, and interstitial inflammation. Briefly, a score of 0 was assigned to kidneys that exhibited minimal changes within a normal range for 6 -month-old rats. Score 1 was assigned to kidneys that exhibited morphologic changes in approximately $25 \%$ glomeruli, up to $10 \%$ tubules, and contained focal small interstitial infiltrate composed of mononuclear inflammatory cells. Score 2 was assigned to kidneys that exhibited involvement of approximately $50 \%$ glomeruli, up to $20 \%$ tubules, and contained multiple small foci of inflammatory cells within the interstitium. Grade 3 was assigned to kidneys that showed involvement of approximately $75 \%$ glomeruli, up to $30 \%$ tubules, and contained medium-sized aggregate of inflammatory cells within the interstitium. Grade 4 was assigned to kidneys that exhibited morphologic changes in more than $75 \%$ glomeruli, up to $40 \%$ tubules, and contained relatively medium-sized aggregates of inflammatory cells along with the interstitial fibrosis. Grade 5 was assigned when more than $40 \%$ tubules exhibited morphologic changes. Model-related unilateral or bilateral hydronephrosis was observed in multiple animals. Sections exhibiting hydronephrotic changes were excluded from 


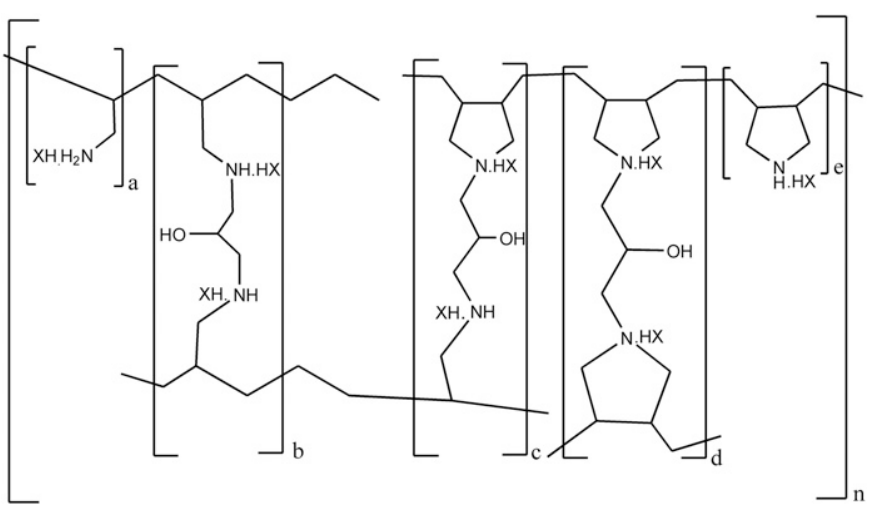

Fig. 1. Representative structure of SAR442357. X is the counter anion of the acid, in this case HCO3- (bicarbonate)

histopathological assessment, such as sections from one obese ZSF1 rat treated with colesevelam $(2 \%)$.

\section{Statistical Analysis}

Results are represented as means \pm S.D. or medians \pm range for histologic parameters. Statistical analysis (GraphPad Prism software) for treatment effects versus the obese ZSF1 control group was performed using GraphPad Prism (GraphPad Prism Software Inc., San Diego, CA) with two-way ANOVA followed by Dunnett's multiple comparisons test. $P \leq 0.05$ was considered significant.

Gene expression values are represented as fold change for treatment groups versus the obese ZSF1 control group. The variable multiplicity was considered, and false discovery rate (FDR)-adjusted $P$ values were calculated using the Benjamini-Hochberg correction (Benjamini and Hochberg, 1995). FDR $\leq 0.05$ was considered significant.

Kidney weight and histologic values were analyzed for normality with Kolmogorov-Smirnov test and subsequently with one-way ANOVA for treatment effects versus the obese ZSF1 control group with Kruskal-Wallis test. $P \leq 0.05$ was considered significant.

\section{Results}

\section{Synthesis and Characterization of Sequestrant Polymers}

Sequestrants of phosphate and bile salts represent a class of amine-containing (cationic) water-swellable polymer networks. These polymer networks, commonly addressed as hydrogels, are obtained by covalent crosslinking of soluble polymer chains. The crosslinking process renders the polymers to be nonabsorbable from the GI tract. By remaining confined to GI tract without systemic exposure, these hydrogels bind target anions in the gut, primarily via electrostatic interactions. SAR442357 is a structurally optimized amine functionalized copolymer hydrogel, which is obtained by crosslinking of PAA and PDA using epichlorohydrin as the crosslinker. Figure 1 represents the schematic structure of a representative portion of SAR442357. This polymer was identified through a parallel synthesis process. Systematic optimization of the physicochemical properties was pursued using a design of experiment strategy in which the effects of variation of experimental parameters such as relative amounts of PAA, PDA, and epichlorohydrin in the polymer hydrogels were assessed. Toward that end, a library of 21 polymer hydrogels of different compositions were synthesized. The resulting hydrogels were characterized by using different physical and chemical techniques. The results of characterization and in vitro substrate binding properties of these hydrogels are summarized in the supporting information (Supplemental Table 1). High-resolution two-dimensional solid-state NMR spectroscopy was used to systematically characterize the molecular structure of SAR442357. In particular, two-dimensional ${ }^{1} \mathrm{H}-{ }^{13} \mathrm{C}$ correlation NMR spectroscopy (Supplemental Fig. 1) enabled us to unequivocally assign the structure of composition of this polymer.

Relative ratios of PAA and PDA contents in the hydrogel can lead to distinct spatial distribution of the amine functionalities across the polymer network that may influence the electrostatic interaction with the anionic substrates. On the other hand, the amount of epichlorohydrin modulates the physical properties of the hydrogels, including ease of particle formation and swelling properties. With the appropriate degree of crosslinking, hydrogels will exhibit adequate availability of binding sites for the electrostatic interactions with the substrates in the gut without significant increase in swelling. Furthermore, the particle size of the resulting hydrogel has significant influence on its binding behavior, which can be explained by differences in diffusion rates of the substrates across the hydrogel matrix as a function of particle size.

The in vitro phosphate and bile acid binding properties of these hydrogels were assessed under equilibrium conditions in a physiologically relevant buffer. GC and GCDC were used as representative bile acids for in vitro binding experiments. Based on optimized physicochemical properties and in vitro substrate binding properties (Supplemental Table 1), SAR4422357 was selected as the lead sequestrant for further pharmacological evaluation.

The key binding parameters for colesevelam, the two homopolymers of PAA and PDA, and SAR4422357 are shown in Table 1. The chemical structure of colesevelam comprises an epichlorohydrin crosslinked PAA backbone with pendant hydrophobic alkyl chains tethered to the amine groups of PAA (Steinmetz and Schonder, 2005). SAR4422357 maintains the good substrate binding properties of the PAA homopolymer hydrogel, yet with a lower swell index. The favorable swelling index of SAR4422357 compared with the corresponding homopolymer hydrogels PAA and PDA is expected to reduce potential swelling-mediated undesired GI side effects.

\section{Body Weight and Food Efficiency}

The lean ZSF1 control group had, over the full 3-month period of the study (from 10 to 24 weeks of age), lower body weight compared with the obese ZSF1 control group.

Three months of treatment of the obese ZSF1 rats with SAR442357 (2\% or $4 \%$ ) did not change body weight compared with the obese ZSF1 control group (Fig. 2A), although food intake was higher with SAR442357 4\% from 2 months onward and with SAR442357 $2 \%$ also at 3 months (Fig. 2B). No differences were observed in food efficiency (Fig. 2C). However, energy metabolized (Fig. 2D) and energy loss via feces were higher from 2 months onward for 4\% SAR442357 treatment group and in the $2 \%$ SAR442357 (Fig. 2E) at 3 months compared with the obese ZSF1 control group. No differences were observed in energy loss via urine (Fig. 2F) between the SAR442357 treatment groups and the obese ZSF1 control group.

The lean ZSF1 control group exhibited higher food intake at 3 months and higher food efficiency and metabolized energy from 2 months onward compared with the obese ZSF1 control 
group. Energy loss via feces and urine was consistently low at all measured time points (Fig. 2, E and F).

No changes in body weight, food intake, food efficiency, metabolized energy, or energy loss via urine were observed for the obese ZSF1 rats treated with losartan $(10 \mathrm{mg} / \mathrm{kg})$ or $2 \%$ colesevelam mixed in the diet (Fig. 2, A-D, F). Regarding energy loss via feces, only the obese ZSF1 colesevelam-treated (2\%) group showed higher energy loss from 2 months onward, similar to the SAR442357-treated groups, compared with the obese ZSF1 control group (Fig. 2E).

\section{Diabetes Progression}

Throughout the study period, the lean ZSF1 control group exhibited lower circulating insulin, random glucose, and $\mathrm{HbA} 1 \mathrm{c}$ (\%) levels compared with the obese ZSF1 control group (Fig. 3).

Treatment of obese ZSF1 rats with SAR442357 (2\% or 4\%) for 3 months delayed diabetes progression as indicated by higher circulating insulin (Fig. 3A) and lower glucose (Fig. 3B) levels compared with the obese ZSF1 control group. Furthermore, SAR442357 (2\% or $4 \%$ ) reduced HbA1c (\%) starting at 2 months of treatment (Fig. 3C). These effects were observed at an earlier time point with the highest dose of SAR442357 (4\%), as insulin levels were higher and glucose values lower after 2 months of treatment compared with the ZSF1 obese control group.

The losartan $(10 \mathrm{mg} / \mathrm{kg})$ group did not show differences in these metabolic parameters compared with the obese control group. In the colesevelam (2\%) group, a statistically significant lowering of $\mathrm{HbA} 1 \mathrm{c}(\%)$ values was noted at 2 months (Fig. 3C). The HbA1c (\%) value at 3 months was lower than obese ZSF1 controls but did not reach statistical significance.

\section{Bile Acids and Free Fatty Acids}

Sequestration of bile acids by SAR442357 was assessed by measuring fecal and urinary bile acid levels along the treatment period. Fecal bile acids were lower in the lean ZSF1 control group from 2 months onward, and urine bile acids were lower at all measured time points compared with the obese ZSF1 control group (Fig. 4, A and B). Fecal free fatty acids were also lower at all measured time points (Fig. 4C).

In the obese ZSF1 rats treated with $2 \%$ colesevelam or SAR442357 (2\% or 4\%), fecal bile acids levels were consistently higher in a dose-independent manner from 2 months onward compared with the obese ZSF1 control group (Fig. 4A). However, regarding urine bile acids, only $2 \%$ colesevelam showed a transient decrease in urinary bile acids at 2 months compared with the obese ZSF1 control group (Fig. 4B).

Fecal free fatty acids showed a profile parallel to fecal bile acids, with higher levels in the obese ZSF1 rats treated with $2 \%$ colesevelam and SAR442357 (2\% or $4 \%)$ in a doseindependent manner from 2 months onward compared with the obese ZSF1 control group (Fig. 4C).

Losartan $(10 \mathrm{mg} / \mathrm{kg})$ had no effect on bile acids or free fatty acid levels (Fig. 4).

\section{Serum Triglycerides and Total Cholesterol}

Over the course of the study, the lean ZSF1 control group showed lower serum triglycerides and total cholesterol levels than the obese ZSF1 control group (Fig. 5).

None of the therapeutic agents had any impact on serum triglyceride levels (Fig. 5A). On the other hand, serum cholesterol 
levels were lower after 2 months of treatment with $2 \%$ colesevelam and 4\% SAR442357 compared with the obese ZSF1 control group. However, this effect on cholesterol waned after 3 months with $2 \%$ colesevelam. In comparison, both losartan $(10 \mathrm{mg} / \mathrm{kg})$ and SAR442357 (2\% or $4 \%$ ) decreased serum cholesterol at 3 months, with $4 \%$ SAR442357 having an effect from 2 months onward (Fig. 5B).

\section{Hepatic Gene Expression for Cholesterol, Bile Acids, and Fatty Acid Synthesis Pathways}

To test for hepatic effects of the different treatments, ribonucleic acid sequence analysis was performed on liver samples taken at study termination. The global number of significantly regulated genes for the different treatment groups compared with the obese ZSF1 control group is illustrated in volcano plots in Supplemental Fig. 2. Genes that were downregulated in the obese ZSF1 control group and recovered upon treatment with either colesevelam or SAR442357 are summarized in a heat map (Supplemental Fig. 3). In line with the expected mechanism of action, hepatic mRNA levels for marker genes belonging to cholesterol (Hmgcr, Mvd, Sqle), bile acids (Cyp7a1), and fatty acid (Acly, Acaca, Acacb, Fasn) synthesis pathways were higher in those animals treated with colesevelam (2\%) and SAR442357 (2\% or $4 \%)$ compared with the obese ZSF1 control group (Fig. 6). In all cases, there was a trend toward higher levels with SAR442357 (4\%) compared with SAR442357 (2\%) that was, however, not statistically significant. Losartan treatment had no effect on expression of these genes.

\section{Inorganic Phosphate}

Sequestration of dietary phosphate by SAR442357 was assessed by measuring fecal, urinary, and serum phosphate levels during the treatment period. Fecal and urine inorganic phosphate was lower in the lean ZSF1 control group at all measured time points and serum inorganic phosphate from 2 months on compared with the obese ZSF1 control group (Fig. 7).

Although fecal inorganic phosphate levels were higher in the obese ZSF1 rats treated with colesevelam $(2 \%)$ or SAR442357 (2\% or $4 \%$ ) after 2 months compared with the obese ZSF1 control group, these differences only persisted with colesevelam (2\%) treatment at 3 months (Fig. 7A). However, urine inorganic phosphate levels were low from 2 months onward with SAR442357 (2\% or $4 \%$ ) in a dosedependent manner compared with the obese ZSF1 control group $(P<0.05)$. After 3 months, treatment with colesevelam (2\%) led to lower urinary inorganic phosphate levels (Fig. 7B).

Only treatment with SAR442357 (2\%) led to a transitory decrease in serum inorganic phosphate at 2 months compared with the obese ZSF1 control group (Fig. 7C).

Treatment with losartan $(10 \mathrm{mg} / \mathrm{kg})$ had no effect on inorganic phosphate levels in any of the biologic samples analyzed.

\section{Diabetic Nephropathy Progression}

Urine and Serum Parameters. Throughout the course of the study, the lean ZSF1 control group had lower urine volume, glucose, total protein, albumin/creatine ratio, urinary KIM1, and cystatin C compared with the obese ZSF1 control group (Fig. 8).

Urinary volume and glucose were decreased only with SAR442357 (4\%) after 3 months of treatment compared with the obese ZSF1 control group (Fig. 8, A and B). SAR442357 (4\%) decreased urinary total protein from 2 months onward. Similarly, after 3 months, both losartan $(10 \mathrm{mg} / \mathrm{kg})$ and SAR442357 (2\%) decreased urine total protein compared with

\begin{tabular}{|ll|}
\hline Lean ZSF1 & Obese ZSF1 Colesevelam (2\%) \\
Obese ZSF1 & $\square$ Obese ZSF1 SAR442357 (2\%) \\
Obese ZSF1 Losartan (10 mg/kg/d) & $\square$ Obese ZSF1 SAR442357 (4\%) \\
\hline
\end{tabular}

A
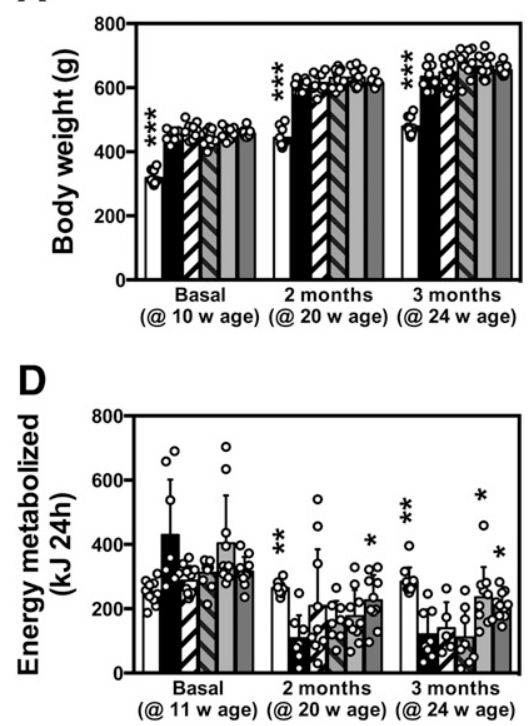

B

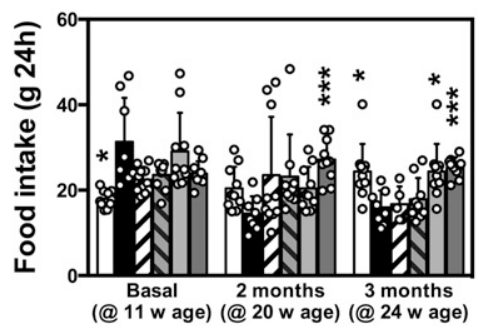

E

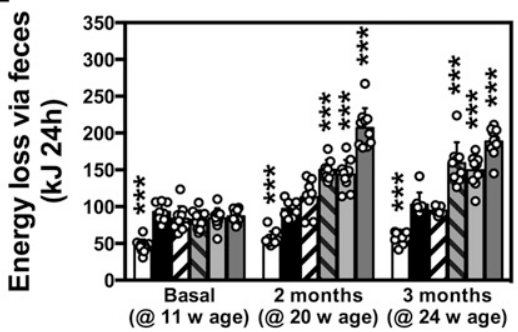

C

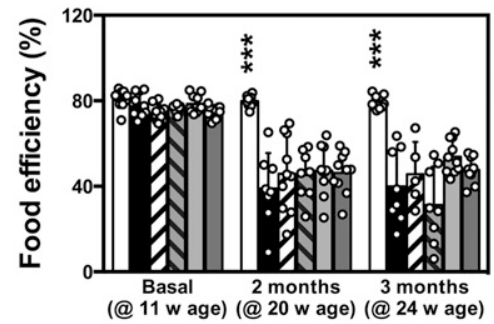

$\mathbf{F}$

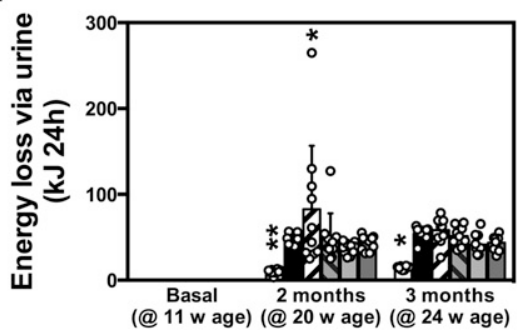

Fig. 2. Body weight (A) and food efficiency (C). Further daily parameters analyzed were food intake (B), metabolized energy (D) and energy loss either via feces $(\mathrm{E})$ or via urine $(\mathrm{F})$. Values are represented as means \pm S.D. Treatment effect versus the obese ZSF1 control group analyzed with two-way ANOVA with Dunnett's multiple comparisons test for statistical differences; $* P<0.05, * * P<0.01$, $* * * P<0.001$ (lean ZSF1 $n=10$, obese ZSF1 $n=8$, obese ZSF1 losartan $n=5-10$, obese ZSF1 colesevelam $n=8-10$, obese ZSF1 SAR442357 2\% $n=10$, obese ZSF1 SAR442357 $4 \% n=10$ ). NM, not measured. 


\begin{tabular}{|lll|}
\hline$\square$ & Lean ZSF1 & Obese ZSF1 Colesevelam (2\%) \\
Obese ZSF1 & $\square$ Obese ZSF1 SAR442357 (2\%) \\
Obese ZSF1 Losartan (10 mg/kg/d) & $\square$ Obese ZSF1 SAR442357 (4\%) \\
\hline
\end{tabular}

A

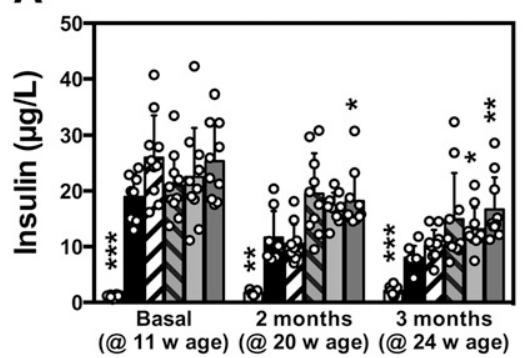

B

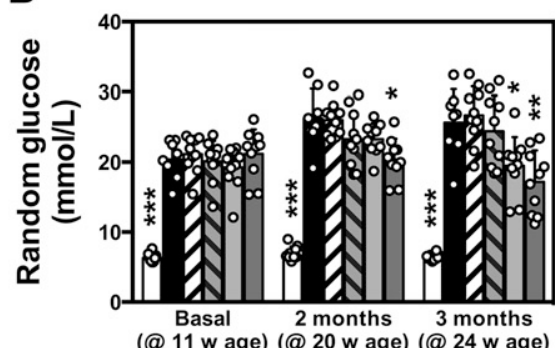

C

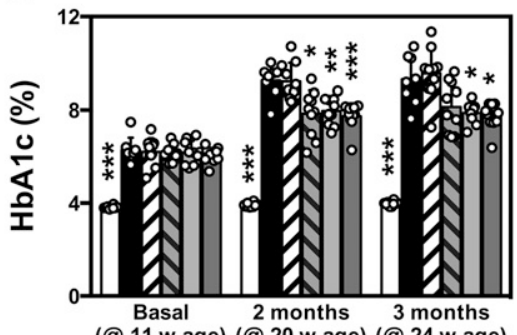

(@11 w age) (@20 w age) (@ 24 w age)

Fig. 3. Diabetes progression as observed on the blood parameters insulin (A), random fed blood glucose (B) and HbA1c (C). Values are represented as means \pm S.D. Treatment effect vs. the obese ZSF1 control group analyzed with two-way ANOVA with Dunnett's multiple comparisons test for statistical differences; $* P<0.05, * * P<0.01$, **** $P<0.001$ (lean ZSF1 $n=10$, obese ZSF1 $n=8$, obese ZSF1 losartan $n=10$, obese ZSF1 colesevelam $n=10$, obese ZSF1 SAR442357 2\% $n=9$ to 10, obese ZSF1 SAR442357 4\% $n=10$ ).

the obese ZSF1 control group (Fig. 8C). Colesevelam (2\%) had no effects on urinary total protein.

Urinary albumin/creatinine ratio was decreased from 2 months onward with losartan $(10 \mathrm{mg} / \mathrm{kg})$ and SAR442357 (4\%) and at 3 months with SAR442357 (2\%) compared with the obese ZSF1 control group. Colesevelam (2\%) decreased the albumin/creatinine ratio transiently at 2 months (Fig. 8D).

Losartan $(10 \mathrm{mg} / \mathrm{kg})$ and SAR442357 (4\%) decreased urine KIM1 from 2 months onward compared with the obese ZSF1 control group (Fig. 8E).

SAR442357 (4\%) decreased urine cystatin C from 2 months onwards, and at 3 months, both losartan $(10 \mathrm{mg} / \mathrm{kg})$ and SAR442357 (2\%) decreased cystatin C compared with the obese ZSF1 control group (Fig. 8F).

In addition to the urinary kidney disease progression markers measured throughout the course of the study, we measured kidney weights (Fig. 9) and final concentrations of the biomarkers attributed to chronic tubular damage serum urea and serum creatinine (Supplemental Fig. 7) at the end of the study.

Kidney weights were decreased only with SAR442357 ( $2 \%$ or $4 \%$ ) after 3 months of treatment compared with the obese ZSF1 control group (Fig. 9).
Serum urea concentration was significantly lower in lean ZSF1 rats compared with the obese ZSF1 control animals. Furthermore, it significantly decreased in obese ZSF1 rats treated with losartan $(10 \mathrm{mg} / \mathrm{kg})$ and SAR442357 (4\%) (Supplemental Fig. 7A). On the other hand, no differences in serum creatinine values were observed between the treated groups and the control group in obese ZSF1 rats (Supplemental Fig. 7B).

Histopathological Findings. Rats treated with $4 \%$ SAR442357 showed a significant $(P<0.05)$ decrease in the progression of renal injury, including glomerular pathology, tubular intraluminal protein casts, and tubular pathology (Figs. 10 and 11; Supplemental Fig. 6). Although statistically insignificant, improvement in interstitial inflammation was also observed in rats treated with 4\% SAR442357. In comparison, obese ZSF1 control rats showed most severe changes in the glomerular and tubular compartments along with the segmental and multifocal tubulointerstitial areas. Glomerular changes included mesangial thickening by periodic acid-Schiff (PAS)-stained material, synechiae and crescent formation, splitting and thickening of Bowman's capsule, thickened capillary walls and lower luminal diameter, lobulation of glomerular tufts, obliteration of

\begin{tabular}{|ll|}
\hline$\square$ Lean ZSF1 & Obese ZSF1 Colesevelam (2\%) \\
Obese ZSF1 & $\square$ Obese ZSF1 SAR442357 (2\%) \\
Oabese ZSF1 Losartan (10 mg/kg/d) & $\square$ Obese ZSF1 SAR442357 (4\%) \\
\hline
\end{tabular}

A

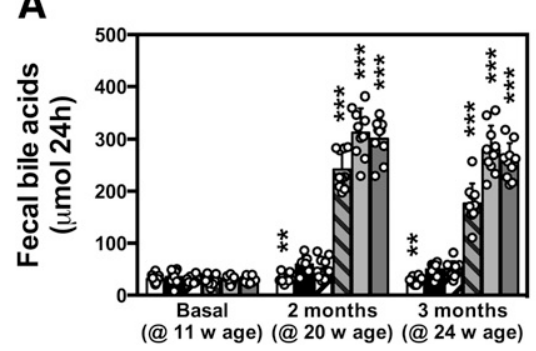

B

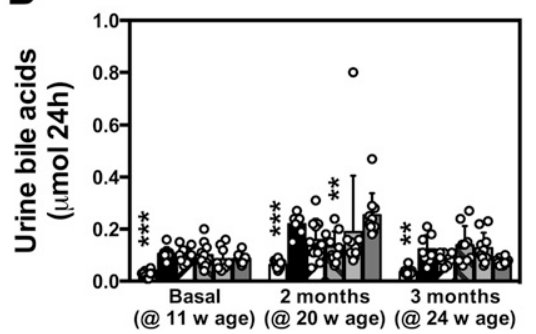

C

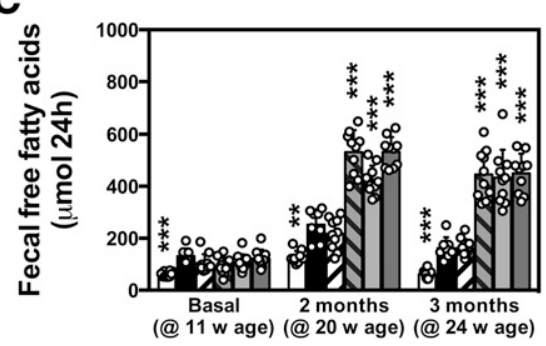

Fig. 4. Fecal (A) and urine (B) bile acids and fecal free fatty acids (C). Values are represented as means \pm S.D. Treatment effect vs. the obese ZSF1 control group analyzed with two-way ANOVA with Dunnett's multiple comparisons test for statistical differences; $* P<0.05$, $* * P<0.01, * * * P<0.001$ (lean ZSF1 $n=10$, obese ZSF1 $n=8$, obese ZSF1 losartan $n=10$, obese ZSF1 colesevelam $n=9$ to 10 , obese ZSF1 SAR442357 $2 \% n=9$ to 10 , obese ZSF1 SAR442357 $4 \% n=10)$. 


\begin{tabular}{|ll|}
\hline$\square$ Lean ZSF1 & Obese ZSF1 Colesevelam (2\%) \\
Obese ZSF1 & $\square$ Obese ZSF1 SAR442357 (2\%) \\
Obese ZSF1 Losartan (10 mg/kg/d) & $\square$ Obese ZSF1 SAR442357 (4\%) \\
\hline
\end{tabular}

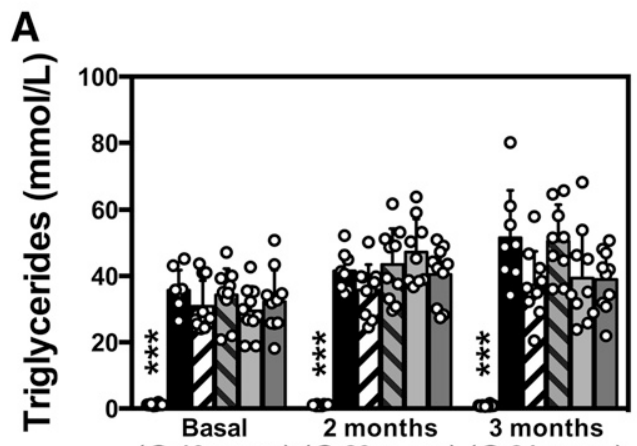

(@10w age) (@20w age) (@ 24 w age)
B

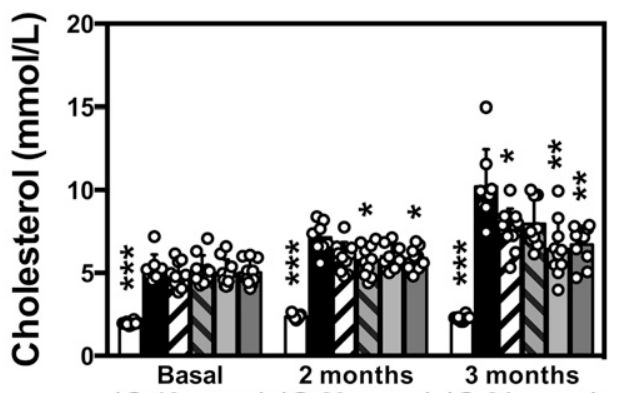

(@10 w age) (@20 w age) (@ 24 w age)

Fig. 5. Serum triglycerides (A) and total cholesterol (B). Values are represented as means \pm S.D. Treatment effect vs. the obese ZSF1 control group analyzed with two-way ANOVA with Dunnett's multiple comparisons test for statistical differences; ${ }^{*} P<0.05, * * P<0.01$, ${ }^{* * *} P<0.001$ (lean ZSF1 $n=10$, obese ZSF1 $n=8$, obese ZSF1 losartan $n=10$, obese ZSF1 colesevelam $n=9$ to 10 , obese ZSF1 SAR442357 $2 \% n=9$ to 10 , obese ZSF1 SAR442357 $4 \% n=10$ ).

glomerular spaces with eosinophilic material, and regionalto-global obsolescence. Tubulointerstitial changes included dilation of cortical and medullary tubules, presence of intraluminal protein casts, thickened tubular basement membrane, tubular epithelial cell degeneration, cortical perivascular aggregates of mononuclear inflammatory cells, and regional interstitial collapse with tubular atrophy and fibrosis (Fig. 11). Compared with 4\% SAR442357, rats treated with losartan (10 mg/kg per day) showed progression of glomerular pathology, whereas the treatment effects on assessed

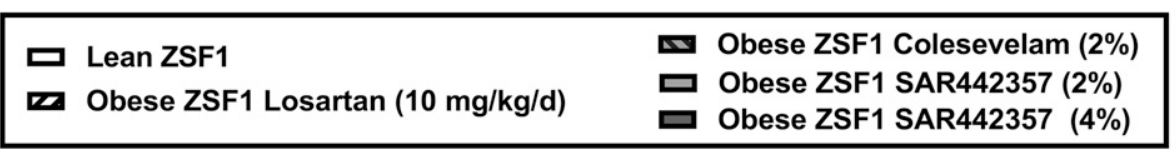

A

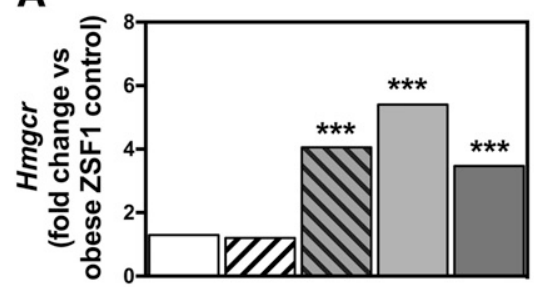

D

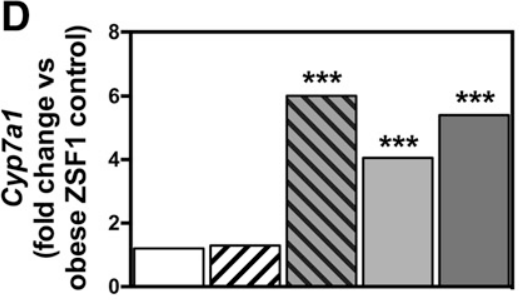

G

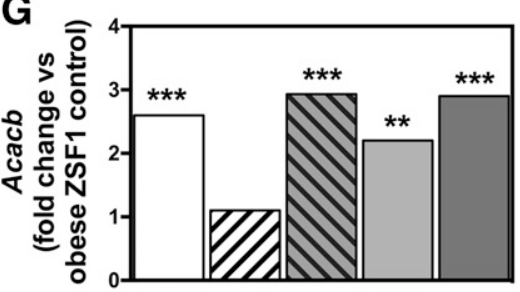

B

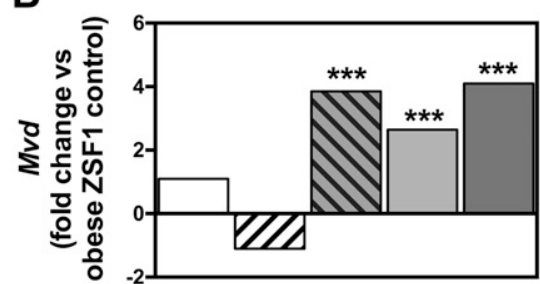

E

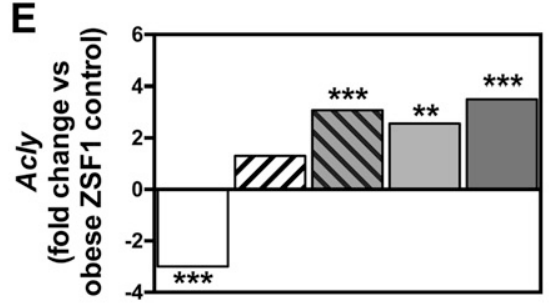

H

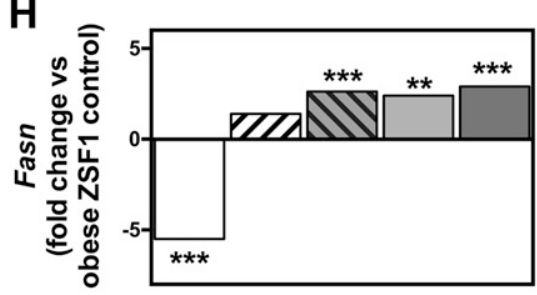

C

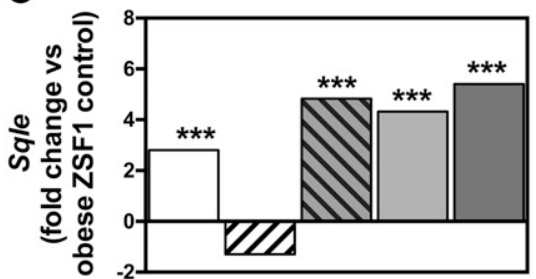

$F$

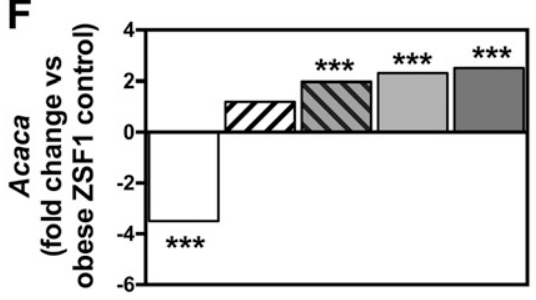

I

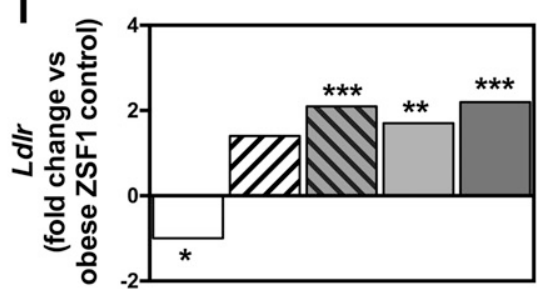

Fig. 6. Hepatic gene expression for cholesterol (A, B, C), bile acids (D), fatty acid synthesis (E, F, G, H) and cholesterol endocytosis (I) pathways. Values represented as fold change vs. the obese ZSF1 control group. Analysis with false discovery rate with Benjamini-Hochberg correction for multiplicity for statistical differences; *FDR $<0.05$, **FDR $<0.01$, ***FDR $<0.001$ (lean ZSF1 $n=10$, obese ZSF1 $n=8$, obese ZSF1 losartan $n=10$, obese ZSF1 colesevelam $n=10$, obese ZSF1 SAR442357 2\% $n=10$, obese ZSF1 SAR442357 4\% $n=10$ ). 3-hydroxy-3-methylglutaryl-coenzyme A reductase (Hmgcr); Mevalonate diphosphate decarboxylase (Mvd); Squalene monooxygenase (Sqle); ATP citrate lyase (Acly)Acetyl-CoA carboxylase alpha (Acaca); AcetylCoA carboxylase beta (Acacb)Fatty acid synthase (Fasn) 

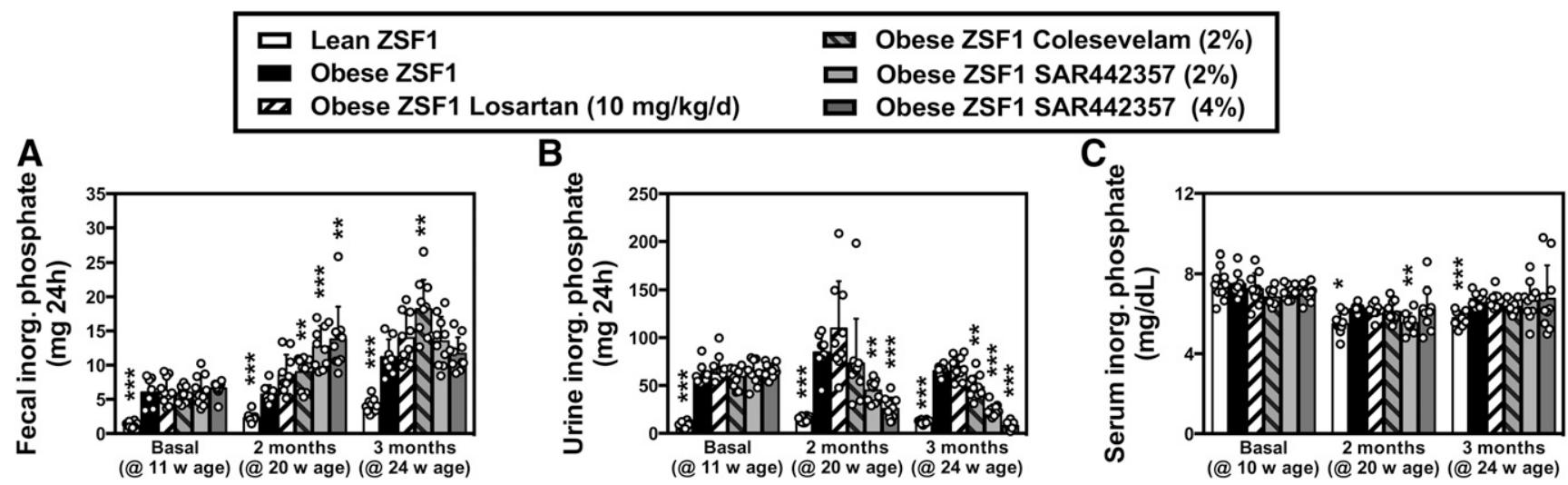

Fig. 7. Fecal (A), urine (B) and serum (C) inorganic phosphate. Values are represented as means \pm S.D. Treatment effect vs. the obese ZSF1 control group analyzed with two-way ANOVA with Dunnett's multiple comparisons test for statistical differences; $* P<0.05$, $* * P<0.01, * * * P<0.001$ (lean ZSF1 $n=9$ to 10 , obese ZSF1 $n=8$, obese ZSF1 losartan $n=9$ to 10 , obese ZSF1 colesevelam $n=9$ to 10 , obese ZSF1 SAR442357 $2 \% n=9$ to 10 , obese ZSF1 SAR442357 $4 \% n=9$ to 10$)$.

parameters such as tubular protein casts, tubular pathology, and interstitial inflammation were comparable (Figs. 10 and 11; Supplemental Fig. 6). Similarly, rats treated with $2 \%$ colesevelam exhibited no improvement in glomerular pathology, tubular protein casts, and tubular pathology parameters when compared with the treatment with $4 \%$ SAR442357 (Figs. 10 and 11; Supplemental Fig. 6).

\section{Discussion}

The goal of this investigation was to evaluate pharmacological benefits of a nonabsorbed polymeric sequestrant
(SAR442357) that possesses optimum phosphate and bile acid binding characteristics in attenuating the progression of DKD and preventing associated cardiovascular complications in T2D using a relevant preclinical animal model. Our findings suggest that treatment of obese ZSF1 rats with SAR442357 for 3 months may attenuate the progression of DKD in a manner similar to losartan, an angiotensin II type 1 receptor blocker approved for this purpose. On the other hand, use of colesevelam, a clinically approved BAS (with poor phosphate binding characteristics) did not offer any therapeutic benefits for DKD. However, unlike losartan, both colesevelam and SAR442357 improved glycemia in these animals. This was evident from an

\begin{tabular}{|ll|}
\hline$\square$ Lean ZSF1 & Obese ZSF1 Colesevelam (2\%) \\
Obese ZSF1 & $\square$ Obese ZSF1 SAR442357 (2\%) \\
Obese ZSF1 Losartan (10 mg/kg/d) & $\square$ Obese ZSF1 SAR442357 (4\%) \\
\hline
\end{tabular}

A

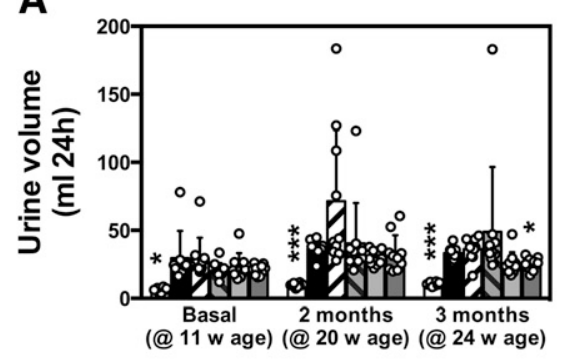

D

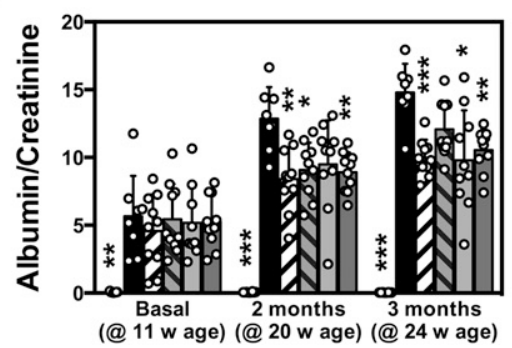

B

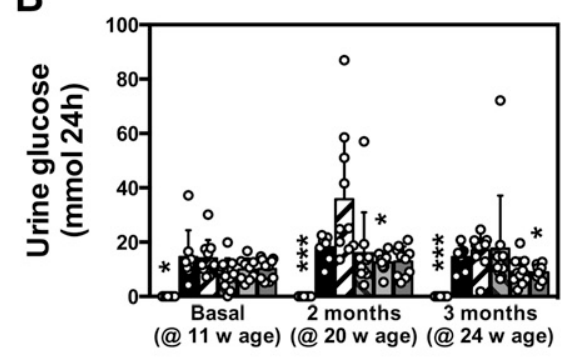

E

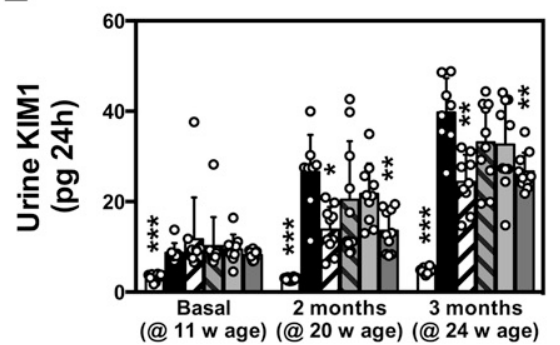

C

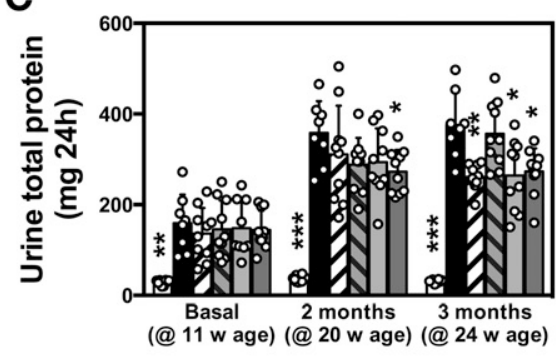

$\mathbf{F}$

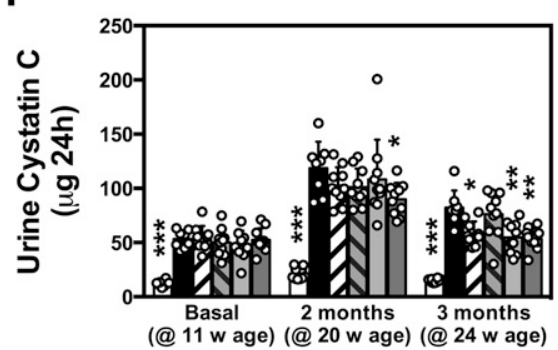

Fig. 8. Diabetic nephropathy progression as observed on the urinary parameters volume (A), glucose (B), total protein (C), albumine/creatinine ratio (D) as well as the biomarkers KIM1 (E) and cystacin (F). Values are represented as means \pm S.D. Treatment effect vs. the obese ZSF1 control group analyzed with two-way ANOVA with Dunnett's multiple comparisons test for statistical differences; $* P<0.05, * * P<0.01$, *** $P<0.001$ (lean ZSF1 $n=10$, obese ZSF1 $n=8$, obese ZSF1 losartan $n=9$ to 10 , obese ZSF1 colesevelam $n=9$ to 10 , obese ZSF1 SAR442357 $2 \% n=9$ to 10 , obese ZSF1 SAR442357 $4 \% n=10)$. 


\begin{tabular}{|ll|}
\hline 미 Lean ZSF1 & Obese ZSF1 Colesevelam (2\%) \\
Obese ZSF1 & 口 Obese ZSF1 SAR442357 (2\%) \\
\hline Obese ZSF1 Losartan (10 mg/kg/d) & 口 Obese ZSF1 SAR442357 (4\%) \\
\hline
\end{tabular}

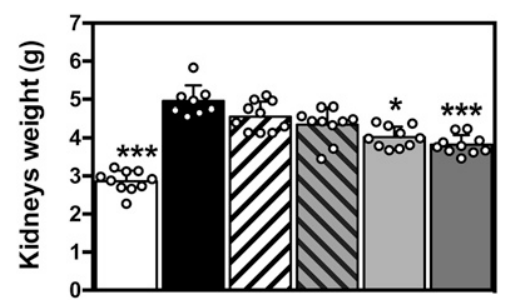

Fig. 9. Kidney weight. Values are represented as means \pm S.D. Treatment effect vs. the obese ZSF1 control group analyzed with one-way ANOVA on ranks (Kruskal-Wallis test) for statistical differences; $* P<0.05$, $* * * P<0.001$ (lean ZSF1 $n=10$, obese ZSF1 $n=8$, obese ZSF1 losartan $n=10$, obese ZSF1 colesevelam $n=10$, obese ZSF1 SAR442357 $2 \% n=10$, obese ZSF1 SAR442357 $4 \% n=10$ ).

increase in plasma insulin and decrease in plasma glucose and HbA1c in the colesevelam- or SAR442357-treated animals.

The pharmacological actions of lumen-restricted BASs stem from their abilities to sequester and remove bile acids from the intestinal lumen, thereby decreasing their enterohepatic circulation. This process leads to increased hepatic synthesis of new bile acids from cholesterol, which in turn increases the requirement for cholesterol in the liver. This results in increased uptake of cholesterol via the low density lipoprotein receptor pathway and an overall decrease in blood cholesterol. Moreover, BASs such as colesevelam have been shown to improve hyperglycemia in preclinical animal models of diabetes (Herrema et al., 2010; Sedgeman et al., 2018) as well as in patients with T2D (Takebayashi et al., 2010; Handelsman, 2011; Nerild et al., 2018). Various mechanisms have been proposed for the antidiabetic properties of BASs, including increased secretion of glucagon-like peptide 1 through activation of Takeda G protein-coupled Receptor 5 (TGR5) and decreased activation of intestinal nuclear farnesoid $\mathrm{X}$ bile acid receptor (Herrema et al., 2010; Takebayashi et al., 2010; Potthoff et al., 2013). Although colesevelam has been reported to improve glycemia in diabetes models such as Zucker Diabetic Fatty (ZDF) (fa/fa) rats and $\mathrm{db} / \mathrm{db}$ mice (Shang et al., 2012; Sedgeman et al., 2018), it has not been evaluated in ZSF1 rats, a model of diabetic kidney disease (Griffin et al., 2007; Homer and Dower, 2018). In the present study, colesevelam exhibited a modest effect on improving glycemic control and progression of renal dysfunction in comparison with untreated obese ZSF1 rats. At similar dose levels, SAR442357 improved glycemia and delayed progression of kidney impairment in the ZSF1 rat model of DKD. In contrast, losartan failed to improve glycemic control as expected (Castoldi et al., 2019) but improved renal decline in ZSF1 rats, as reported previously ( $\mathrm{Su}$ et al., 2018), and its renal effects were comparable to SAR442357 treatment. Improved therapeutic benefits offered by SAR442357 may be attributed to its higher potency at reducing enterohepatic uptake of bile acids compared with colesevelam (Fig. 4A, $P<0.05$ ). These results are supported by lower serum total cholesterol and higher hepatic gene expression of markers for cholesterol and bile acid biosynthesis in the SAR442357-treated group compared with the obese ZSF1 control group. In addition, SAR442357 is a significantly stronger binder of phosphate than colesevelam (Fig. 7). Elevated serum phosphate has emerged as an important cardiovascular risk factor in patients with diabetes and kidney disease. Numerous preclinical and clinical studies have shown that there is a linear correlation between serum phosphate and $\mathrm{T} 2 \mathrm{D}, \mathrm{DKD}$, and coronary artery disease (Hutchison et al., 2011; Streja et al., 2013; Vervloet et al., 2017). Removal of dietary phosphate from the GI tract using phosphate sequestrants has been shown to reduce systemic absorption of phosphate and can lead to improvements in progression of CKD and associated cardiovascular complications. Thus, optimized phosphate and bile acid binding characteristics of SAR442357 may be attributed to its markedly improved therapeutic benefit as compared with colesevelam and losartan.

In terms of chemical structure, SAR442357 is different from colesevelam. The former contains pendant primary and

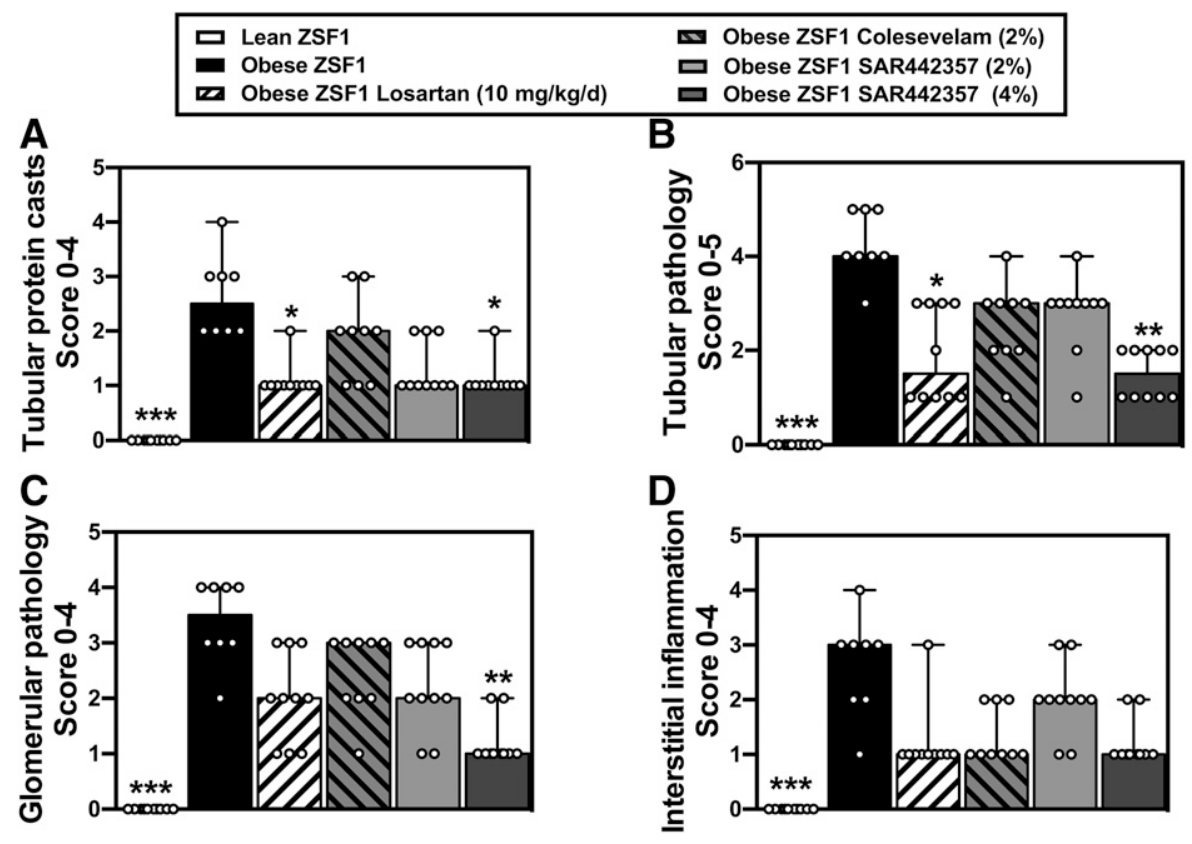

Fig. 10. Kidney histopathology scores for tubular protein casts (A), tubular pathology (B), glomerular pathology (C) and intersticial inflammation (D). Values are represented as medians with range. Treatment effect vs. the obese ZSF1 control group analyzed with one-way ANOVA on ranks (Kruskal-Wallis test) for statistical differences; $* P<0.05, * * P<0.01, * * * P<0.0001$ (lean ZSF1 $n=10$, obese ZSF1 $n=8$, obese ZSF1 losartan $n=9$, obese ZSF1 colesevelam $n=10$, obese ZSF1 SAR442357 $2 \% n=10$, obese ZSF1 SAR442357 $4 \% n=10$ ). 

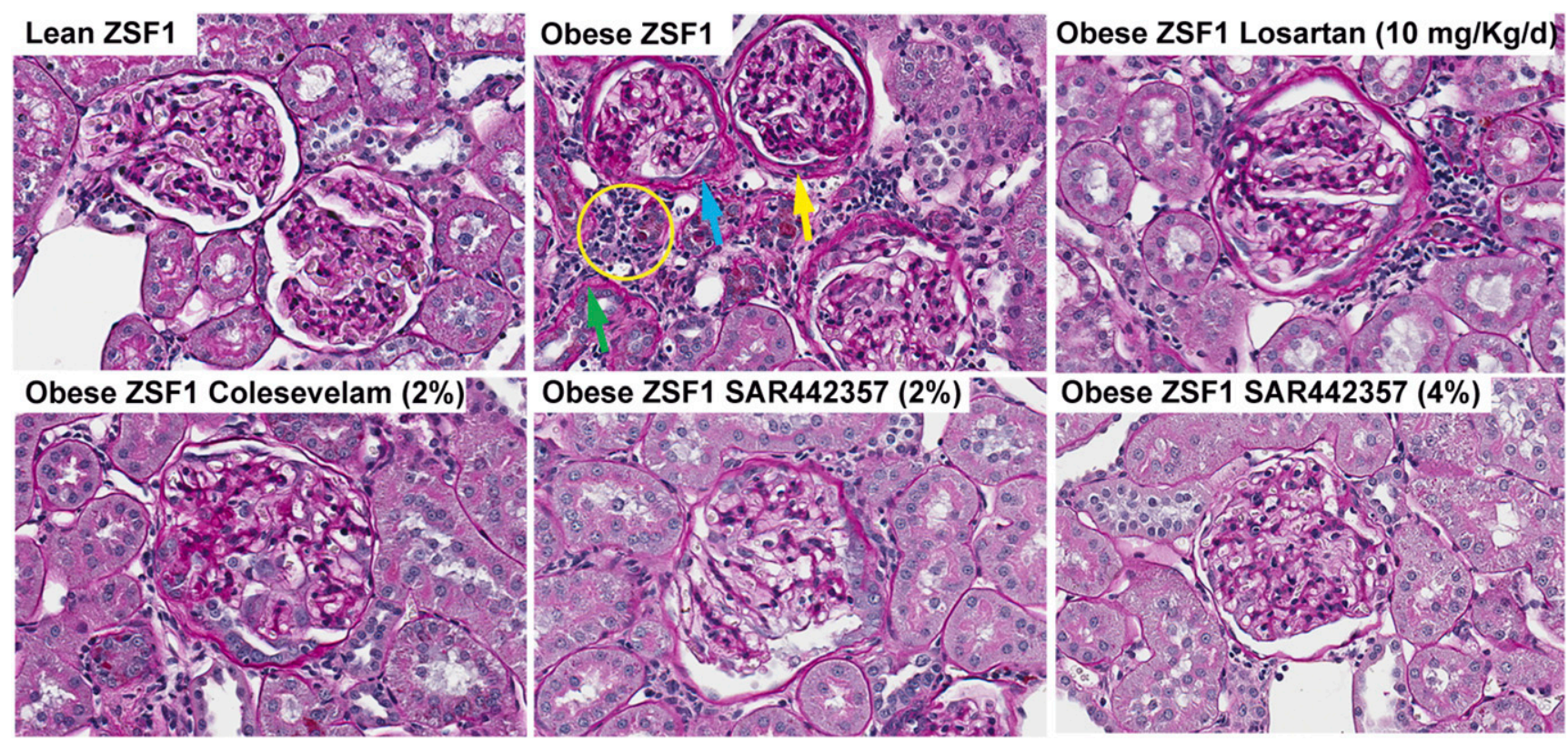

Fig. 11. Kidney histopathology. Compared with the lean ZSF1 rat, kidney from control obese ZSF1 rat shows glomeruli surrounded by a thick PASpositive Bowman's capsule (blue arrow) with regional splitting (yellow arrow), thickened glomerular mesangium, narrow capillary lumena, and synechae formation. Tubules are surrounded by a thick PAS-positive basement membrane (green arrow). Mononuclear inflammatory cells are present in the surrounding interstitium (yellow circle). Losartan-, colesevelam-, and SAR442357-treated rats show reduced glomerular and tubulointerstitial pathology. Note the relative lack of interstitial inflammatory cells in rats treated with colesevelam (2\%) or SAR442357. PAS stain. Magnification, 200 $\times$.

secondary amine groups, with the latter being part of a rigid six-membered ring structure (Fig. 1). On the other hand, in addition to a limited number of primary amine groups, colesevelam contains pendant hydrophobic alkyl chains and quaternary ammonium groups (Steinmetz and Schonder 2005). Presence of higher amounts (millimoles per gram) of primary amine groups in SAR442357 manifests its substantially higher in vitro binding capacity for phosphate ions (5.65 $\mathrm{mmol} / \mathrm{g}$ polymer $)$ compared with colesevelam $(1.29 \mathrm{mmol} / \mathrm{g}$ polymer) (Table 1). In addition, SAR442357 also exhibits higher binding capacity for bile salts. The differences in phosphate binding capacities in vitro between SAR442357 and colesevelam mirror the observed in vivo potencies. Thus, after 2 months of treatment, although SAR442357 treatment resulted in decreased urinary phosphate excretion in a dosedependent manner (Fig. 7B, $P<0.05$ ), urinary phosphate lowering in colesevelam-treated animals was very modest. Improved phosphate lowering effect of SAR442357 can be attributed to its ability to sequester dietary phosphate in the gut with higher binding strength and capacity compared with colesevelam. It may be noted that a clinically approved nonabsorbed polymeric phosphate sequestrant, sevelamer, contains only primary amine groups along its backbone (Burke et al., 2001).

In our study, losartan treatment led to reduced urinary albumin/creatine ratio, total protein, KIM1, cystatin C, and serum total cholesterol in obese ZSF1 rats. These findings are similar to those reported in uninephrectomized ZSF1 rats treated with losartan at the same dose (Su et al., 2018). Increase in urinary KIM1 and cystatin C concentrations are markers for tubular damage and have been used to predict progression of diabetic nephropathy (Conti et al., 2006; Herget-Rosenthal et al., 2007; Kim et al., 2013; Pais et al., 2019; Fernando et al., 2019). Reduction of urinary KIM1 with losartan has also been observed in humans (Nielsen et al.,
2011). However, to our knowledge, the effect of losartan on urinary cystatin $\mathrm{C}$ has only been studied in children with $\mathrm{CKD}$, and the results were inconsistent over 3 years of study (Webb et al., 2012). Similarly, in uninephrectomized ZSF1 rats, after 12 weeks of treatment with losartan, no change in urinary cystatin C was observed (Su et al., 2018). Although decrease in total cholesterol with losartan has been reported in Sprague-Dawley rats fed with a high-fat diet (Mourad et al., 2013), the results have not been translated in humans (Xiao et al., 2016). Furthermore, although losartan has been reported to improve insulin sensitivity in rodents and humans (Mourad et al., 2013; Xiao et al., 2016), in our studies using overtly diabetic ZSF1 rats, it did not show any measurable impact on glycemia. In addition to lowering serum cholesterol, SAR442357 treatment resulted in a decrease in urine volume, urine total protein, albumin/creatinine ratio, KIM1, and cystatin $\mathrm{C}$ and slowed the progression of diabetes. The pharmacological benefits elicited by SAR442357 in improving both DKD and glycemia in obese ZSF1 rats combines those produced by losartan and colesevelam and reported in humans (Brenner et al., 2001; Rosenstock et al., 2010; Xiao et al., 2016).

In summary, our studies showed that developing a novel nonabsorbed polymeric sequestrant with optimum phosphate and bile salt sequestration properties such as SAR442357 may offer combined pharmacological benefits, including glucose regulation, lipid lowering, and attenuation of DKD progression in a single therapeutic agent. These effects can result in overall improvements in kidney disease and pathology, which is evident from lower hypertrophy and the histopathological analysis. Furthermore, it should be noted that polypharmacological characteristics of SAR442357 are similar to those of sodium-dependent glucose cotransporter-2 inhibitor empagliflozin reported in ZSF1 rats (Park et al., 2020). The latter has been approved as a treatment option for patients with CKD/DKD. Taken together, the results presented here 
suggest that SAR442357 exerts a number of pharmacological benefits in improving kidney functions and reducing cardiovascular complications in a rodent model of DKD. Finally, being systemically nonabsorbed, this compound could be a safe and effective drug candidate to treat $\mathrm{DKD}$.

\section{Acknowledgments}

The authors thank Marion Wolf, Elke Kleinschmidt, Dagmar Fenner-Nau, Manfred Schmalz, Daniel Hein, and Jörn Wandschneider for their help with the animal studies; Steve Polomoscanik and Chinyere Agbugba for their help with the polymer synthesis; and Peyman Sakhaii for his help with NMR studies.

\section{Authorship Contributions}

Participated in research design: Castañeda, Konkar, Larsen, Hübschle.

Contributed new reagents or analytic tools: Davison, Schwahn, Scherer, Singh, Dhal.

Performed data analysis: Castañeda, Méndez, Elvert, Boldina, Rocher, Singh, Bangari, Kannt, Dhal, Hübschle.

Wrote or contributed to the writing of the manuscript: Castañeda,

Méndez, Davison, Elvert, Boldina, Rocher, Scherer, Singh, Bangari, Falkenhahn, Kannt, Konkar, Arbeeny, Dhal, Hübschle.

\section{References}

Alicic RZ, Rooney MT, and Tuttle KR (2017) Diabetic kidney disease: challenges, progress, and possibilities. Clin J Am Soc Nephrol 12:2032-2045.

Asmus HG, Braun J, Krause R, Brunkhorst R, Holzer H, Schulz W, Neumayer HH Raggi P, and Bommer J (2005) Two year comparison of sevelamer and calcium carbonate effects on cardiovascular calcification and bone density. Nephrol Dial Transplant 20:1653-1661.

Benjamini Y and Hochberg Y (1995) Controlling the false discovery rate: a practical and powerful approach to multiple testing. JR Stat Soc B 57:289-300.

Bilan VP, Salah EM, Bastacky S, Jones HB, Mayers RM, Zinker B, Poucher SM, and Tofovic SP (2011) Diabetic nephropathy and long-term treatment effects of rosiglitazone and enalapril in obese ZSF1 rats. J Endocrinol 210:293-308.

Bilous R (2008) Microvascular disease: what does the UKPDS tell us about diabetic nephropathy? Diabet Med 25 (Suppl 2):25-29.

Block GA, Hulbert-Shearon TE, Levin NW, and Port FK (1998) Association of serum phosphorus and calcium $\mathrm{x}$ phosphate product with mortality risk in chronic hemodialysis patients: a national study. Am J Kidney Dis 31:607-617.

Block GA, Klassen PS, Lazarus JM, Ofsthun N, Lowrie EG, and Chertow GM (2004) Mineral metabolism, mortality, and morbidity in maintenance hemodialysis. $J$ Am Soc Nephrol 15:2208-2218.

Block GA, Raggi P, Bellasi A, Kooienga L, and Spiegel DM (2007) Mortality effect of coronary calcification and phosphate binder choice in incident hemodialysis patients. Kidney Int 71:438-441.

Braunlin W, Zhorov E, Guo A, Apruzzese W, Xu Q, Hook P, Smisek DL, Mandeville WH, and Holmes-Farley SR (2002) Bile acid binding to sevelamer HCl. Kidney Int 62:611-619.

Brenner BM, Cooper ME, de Zeeuw D, Keane WF, Mitch WE, Parving HH, Remuzzi G, Snapinn SM, Zhang Z, and Shahinfar S; RENAAL Study Investigators (2001) Effects of losartan on renal and cardiovascular outcomes in patients with type 2 diabetes and nephropathy. N Engl J Med 345:861-869.

Brønden A, Larsen EL, Karstoft K, Henriksen T, Vilsbøll T, Poulsen HE, and Knop FK (2020) Changes in oxidative nucleic acid modifications and inflammation following one-week treatment with the BAS sevelamer: two randomised, placebocontrolled trials. J Diabetes Complications 34:107446.

Brønden A, Mikkelsen K, Sonne DP, Hansen M, Våben C, Gabe MN, Rosenkilde M, Tremaroli V, Wu H, Bäckhed F, et al. (2018) Glucose-lowering effects and mechanisms of the bile acid-sequestering resin sevelamer. Diabetes Obes Metab 20 1623-1631.

Burke S, Amin N, Incerti C, Plone M, and Watson N (2001) Sevelamer hydrochloride (Renagel), a nonabsorbed phosphate-binding polymer, does not interfere with digoxin or warfarin pharmacokinetics. J Clin Pharmacol 41:193-198.

Caglar K, Yilmaz MI, Saglam M, Cakir E, Acikel C, Eyileten T, Yenicesu M, Oguz Y, Vural A, Carrero JJ, et al. (2008) Short-term treatment with sevelamer increases serum fetuin-a concentration and improves endothelial dysfunction in chronic kidney disease stage 4 patients. Clin J Am Soc Nephrol 3:61-68.

Cameron JS (2006) The discovery of diabetic nephropathy: from small print to centre stage. J Nephrol 19 (Suppl 10):S75-S87.

Castoldi G, di Gioia CRT, Roma F, Carletti R, Manzoni G, Stella A, Zerbini G, and Perseghin G (2019) Activation of angiotensin type 2 (AT2) receptors prevent myocardial hypertrophy in Zucker diabetic fatty rats. Acta Diabetol 56:97-104.

Chertow GM, Burke SK, and Raggi P; Treat to Goal Working Group (2002) Sevelamer attenuates the progression of coronary and aortic calcification in hemodialysis patients. Kidney Int 62:245-252.

Conti M, Moutereau S, Zater M, Lallali K, Durrbach A, Manivet P, Eschwège P, and Loric S (2006) Urinary cystatin C as a specific marker of tubular dysfunction. Clin Chem Lab Med 44:288-291.
Di Iorio B, Bellasi A, and Russo D; INDEPENDENT Study Investigators (2012) Mortality in kidney disease patients treated with phosphate binders: a randomized study. Clin J Am Soc Nephrol 7:487-493.

Di Iorio B, Molony D, Bell C, Cucciniello E, Bellizzi V, Russo D, and Bellasi A; INDEPENDENT Study Investigators (2013) Sevelamer versus calcium carbonate in incident hemodialysis patients: results of an open-label 24-month randomized clinical trial. Am J Kidney Dis 62:771-778.

Dower K, Zhao S, Schlerman FJ, Savary L, Campanholle G, Johnson BG, Xi L, Nguyen V, Zhan Y, Lech MP, et al. (2017) High resolution molecular and histological analysis of renal disease progression in ZSF1 fa/faCP rats, a model of type 2 diabetic nephropathy. PLoS One 12:e0181861.

Drozdz A, Weiner J, Gebczynska Z, and Krasinska M (1975) Some bioenergetic parameters of wild ruminants. Pol Ecol Stud 1:85-101.

Dvir I, Chayoth R, Sod-Moriah U, Shany S, Nyska A, Stark AH, Madar Z, and Arad SM (2000) Soluble polysaccharide and biomass of red microalga Porphyridium sp. alter intestinal morphology and reduce serum cholesterol in rats. Br J Nutr 84: 469-476.

Elvert R, Wille A, Wandschneider J, Werner U, Glombik H, and Herling AW (2013) Energy loss via urine and faeces--a combustive analysis in diabetic rats and the impact of antidiabetic treatment on body weight. Diabetes Obes Metab 15:324-334.

Fernando BNTW, Alli-Shaik A, Hemage RKD, Badurdeen Z, Hettiarachchi TW, Abeysundara HTK, Abeysekara TDJ, Wazil A, Rathnayake S, Gunaratne J, et al (2019) Pilot study of renal urinary biomarkers for diagnosis of CKD of uncertain etiology. Kidney Int Rep 4:1401-1411.

Floege J (2016) Phosphate binders in chronic kidney disease: a systematic review of recent data. J Nephrol 29:329-340.

Floege J and Ketteler M (2004) Vascular calcification in patients with end-stage renal disease. Nephrol Dial Transplant 19 (Suppl 5):V59-V66.

Francischetti EA, de Abreu VG, da Silva Figueiredo LF, Dezonne RS, and Coutinho ESF (2020) Effects of blood pressure lowering agents on cardiovascular outcomes in weight excess patients: a systematic review and meta-analysis. Am J Cardiovasc Drugs 20:447-470 DOI: 10.1007/s40256-019-00393-x.

Fuchs CD, Paumgartner G, Mlitz V, Kunczer V, Halilbasic E, Leditznig N, Wahlström A, Ståhlman M, Thüringer A, Kashofer K, et al. (2018) Colesevelam attenuates cholestatic liver and bile duct injury in $M d r 2^{-/}$mice by modulating composition, signalling and excretion of faecal bile acids. Gut 67:1683-1691.

Garg JP, Chasan-Taber S, Blair A, Plone M, Bommer J, Raggi P, and Chertow GM (2005) Effects of sevelamer and calcium-based phosphate binders on uric acid concentrations in patients undergoing hemodialysis: a randomized clinical trial. Arthritis Rheum 52:290-295.

Giorda CB, Carnà P, Salomone M, Picariello R, Costa G, Tartaglino B, and Gnavi R (2018) Ten-year comparative analysis of incidence, prognosis, and associated factors for dialysis and renal transplantation in type 1 and type 2 diabetes versus nondiabetes. Acta Diabetol 55:733-740.

Goodman WG, Goldin J, Kuizon BD, Yoon C, Gales B, Sider D, Wang Y, Chung J, Emerick A, Greaser L, et al. (2000) Coronary-artery calcification in young adults with end-stage renal disease who are undergoing dialysis. $N$ Engl $J$ Med 342:1478-1483.

Griffin KA, Abu-Naser M, Abu-Amarah I, Picken M, Williamson GA, and Bidani AK (2007) Dynamic blood pressure load and nephropathy in the ZSF1 (fa/fa cp) model of type 2 diabetes. Am J Physiol Renal Physiol 293:F1605-F1613.

Handelsman Y (2011) Role of bile acid sequestrants in the treatment of type 2 diabetes. Diabetes Care 34 (Suppl 2):S244-S250.

Hansen M, Sonne DP, and Knop FK (2014) Bile acid sequestrants: glucose-lowering mechanisms and efficacy in type 2 diabetes. Curr Diab Rep 14:482.

Harada S and Hasegawa S (1984) Homopolymerization of monoallylammonium salts with azo-initiators. Macromol Chem Rapid Commun 5:27-31.

Herget-Rosenthal S, van Wijk JA, Bröcker-Preuss M, and Bökenkamp A (2007) Increased urinary cystatin $\mathrm{C}$ reflects structural and functional renal tubular impairment independent of glomerular filtration rate. Clin Biochem 40:946-951.

Herrema H, Meissner M, van Dijk TH, Brufau G, Boverhof R, Oosterveer MH, Reijngoud DJ, Müller M, Stellaard F, Groen AK, et al. (2010) Bile salt sequestration induces hepatic de novo lipogenesis through farnesoid $\mathrm{X}$ receptor- and liver $\mathrm{X}$ receptor alpha-controlled metabolic pathways in mice. Hepatology 51: $806-816$

Holman RR, Paul SK, Bethel MA, Matthews DR, and Neil HAW (2008) 10-year followup of intensive glucose control in type 2 diabetes. $N$ Engl J Med 359:1577-1589.

Homer BL and Dower K (2018) 41-Week study of progressive diabetic nephropathy in the ZSF1 fa/fa CP rat model. Toxicol Pathol 46:976-977.

Hruska KA, Mathew S, Lund R, Qiu P, and Pratt R (2008) Hyperphosphatemia of chronic kidney disease. Kidney Int 74:148-157.

Hu J, Ge H, Newman M, and Liu K (2012) OSA: a fast and accurate alignment tool for RNA-Seq. Bioinformatics 28:1933-1934.

Hutchison AJ, Smith CP, and Brenchley PE (2011) Pharmacology, efficacy and safety of oral phosphate binders. Nat Rev Nephrol 7:578-589.

Inzucchi SE, Bergenstal RM, Buse JB, Diamant M, Ferrannini E, Nauck M, Peters AL, Tsapas A, Wender R, and Matthews DR (2015) Management of hyperglycemia in type 2 diabetes, 2015: a patient-centered approach: update to a position statement of the American Diabetes Association and the European Association for the Study of Diabetes. Diabetes Care 38:140-149.

Jang T and Rasmussen PG (1998) Synthesis of polyamines with pendant cyanoimidazole units and their electrochemical properties. J Polym Sci A Polym Chem 36: 2619-2629.

Katsiki N, Tsioufis K, Ural D, and Volpe M (2018) Fifteen years of LIFE (Losartan Intervention for Endpoint Reduction in Hypertension)-Lessons learned for losartan an "old dog playing good tricks". J Clin Hypertens (Greenwich) 20:1153-1159.

Kim SS, Song SH, Kim IJ, Jeon YK, Kim BH, Kwak IS, Lee EK, and Kim YK (2013) Urinary cystatin $\mathrm{C}$ and tubular proteinuria predict progression of diabetic nephropathy. Diabetes Care 36:656-661.

Kodera R, Shikata K, Kataoka HU, Takatsuka T, Miyamoto S, Sasaki M, Kajitani N, Nishishita S, Sarai K, Hirota D, et al. (2011) Glucagon-like peptide-1 receptor agonist 
ameliorates renal injury through its anti-inflammatory action without lowering blood glucose level in a rat model of type 1 diabetes. Diabetologia 54:965-978.

Law CW, Chen Y, Shi W, and Smyth GK (2014) voom: precision weights unlock linear model analysis tools for RNA-seq read counts. Genome Biol 15:R29.

Lytvyn Y, Bjornstad P, van Raalte DH, Heerspink HL, and Cherney DZI (2020) The new biology of diabetic kidney disease-mechanisms and therapeutic implications. Endocr Rev 41:202-231.

Meissner M, Herrema H, van Dijk TH, Gerding A, Havinga R, Boer T, Müller M, Reijngoud D-J, Groen AK, and Kuipers F (2011) Bile acid sequestration reduces plasma glucose levels in $\mathrm{db} / \mathrm{db}$ mice by increasing its metabolic clearance rate. PLoS One 6:e24564.

Moe SM and Chen NX (2004) Pathophysiology of vascular calcification in chronic kidney disease. Circ Res 95:560-567.

Mourad AA, Heeba GH, Taye A, and El-Moselhy MA (2013) Comparative study between atorvastatin and losartan on high fat diet-induced type 2 diabetes mellitus in rats. Fundam Clin Pharmacol 27:489-497.

Navarro-González JF, Mora-Fernández C, Muros de Fuentes M, Donate-Correa J, Cazaña-Pérez V, and García-Pérez J (2011) Effect of phosphate binders on serum inflammatory profile, soluble CD14, and endotoxin levels in hemodialysis patients. Clin J Am Soc Nephrol 6:2272-2279.

Nerild HH, Christensen MB, Knop FK, and Brønden A (2018) Preclinical discovery and development of colesevelam for the treatment of type 2 diabetes. Expert Opin Drug Discov 13:1161-1167.

Nielsen SE, Andersen S, Zdunek D, Hess G, Parving HH, and Rossing P (2011) Tubular markers do not predict the decline in glomerular filtration rate in type 1 diabetic patients with overt nephropathy. Kidney Int 79:1113-1118.

Pais GM, Avedissian SN, O'Donnell JN, Rhodes NJ, Lodise TP, Prozialeck WC, Lamar PC, Cluff C, Gulati A, Fitzgerald JC, et al. (2019) Comparative performance of urinary biomarkers for vancomycin-induced kidney injury according to timeline of injury. Antimicrob Agents Chemother 63:e00079-e19.

Park SH, Farooq MA, Gaertner S, Bruckert C, Qureshi AW, Lee HH, Benrahla D, Pollet B, Stephan D, Ohlmann P, et al. (2020) Empagliflozin improved systolic blood pressure, endothelial dysfunction and heart remodeling in the metabolic syndrome ZSF1 rat. Cardiovasc Diabetol 19:19.

Patel L, Bernard LM, and Elder GJ (2016) Sevelamer versus calcium-based binders for treatment of hyperphosphatemia in CKD: a meta-analysis of randomized controlled trials. Clin J Am Soc Nephrol 11:232-244.

Polomoscanik SC, Holmes-Farley SR, Petersen JS, Sacchiero RJ, and Dhal PK (2012) Hydrophobically modified poly(Allylamine) hydrogels containing internal quaternary ammonium groups as cholesterol lowering agents: synthesis, characterization, and biological studies. J Macromol Sci A 49:1011-1021.

Potthoff MJ, Potts A, He T, Duarte JA, Taussig R, Mangelsdorf DJ, Kliewer SA, and Burgess SC (2013) Colesevelam suppresses hepatic glycogenolysis by TGR5mediated induction of GLP-1 action in DIO mice. Am J Physiol Gastrointest Liver Physiol 304:G371-G380.

Raman A, Baum ST, Colman RJ, Kemnitz JW, Weindruch R, and Schoeller DA (2007) Metabolizable energy intake during long-term calorie restriction in rhesus monkeys. Exp Gerontol 42:988-994.

Rastogi A (2013) Sevelamer revisited: pleiotropic effects on endothelial and cardiovascular risk factors in chronic kidney disease and end-stage renal disease. Ther Adv Cardiovasc Dis 7:322-342.

Rodríguez-Osorio L, Zambrano DP, Gracia-Iguacel C, Rojas-Rivera J, Ortiz A, Egido J, and González Parra E (2015) Use of sevelamer in chronic kidney disease: beyond phosphorus control. Nefrologia 35:207-217.

Rosenbaum DP, Petersen JS, Ducharme S, Markham P, and Goldberg DI (1997) Absorption, distribution and excretion of GT31-104, a novel bile acid sequestrant, in rats and dogs after acute and subchronic administration. J Pharm Sci 86:591-595.

Rosenstock J, Fonseca VA, Garvey WT, Goldberg RB, Handelsman Y, Abby SL, Lai Y-L, Jin X, Misir S, Nagendran S, et al. (2010) Initial combination therapy with metformin and colesevelam for achievement of glycemic and lipid goals in early type 2 diabetes. Endocr Pract 16:629-640.

Sedgeman LR, Beysen C, Allen RM, Ramirez Solano MA, Turner SM, and Vickers KC (2018) Intestinal bile acid sequestration improves glucose control by stimulating hepatic miR-182-5p in type 2 diabetes. Am J Physiol Gastrointest Liver Physiol 315:G810-G823.
Shang Q, Liu MK, Saumoy M, Holst JJ, Salen G, and Xu G (2012) The combination of colesevelam with sitagliptin enhances glycemic control in diabetic ZDF rat model. Am J Physiol Gastrointest Liver Physiol 302:G815-G823.

Singh R, Martin BR, Hickey Y, Teegarden D, Campbell WW, Craig BA, Schoeller DA, Kerr DA, and Weaver CM (2009) Comparison of self-reported, measured, metabolizable energy intake with total energy expenditure in overweight teens. Am $J$ Clin Nutr 89:1744-1750.

Steinmetz KL and Schonder KS (2005) Colesevelam: potential uses for the newest bile resin. Cardiovasc Drug Rev 23:15-30.

Streja E, Lau WL, Goldstein L, Sim JJ, Molnar MZ, Nissenson AR, Kovesdy CP, and Kalantar-Zadeh K (2013) Hyperphosphatemia is a combined function of high serum PTH and high dietary protein intake in dialysis patients. Kidney Int Suppl (2011) 3:462-468

Stroeve JH, Brufau G, Stellaard F, Gonzalez FJ, Staels B, and Kuipers F (2010) Intestinal FXR-mediated FGF15 production contributes to diurnal control of hepatic bile acid synthesis in mice. Lab Invest 90:1457-1467.

Su Z, Widomski D, Ma J, Namovic M, Nikkel A, Leys L, Olson L, Salte K, DonnellyRoberts D, Esbenshade T, et al. (2016) Longitudinal changes in measured glomerular filtration rate, renal fibrosis and biomarkers in a rat model of type 2 diabetic nephropathy. Am J Nephrol 44:339-353.

Su Z, Widomski D, Nikkel A, Leys L, Namovic M, Donnelly-Roberts D, Gopalakrishnan M, and McGaraughty S (2018) Losartan improves renal function and pathology in obese ZSF-1 rats. J Basic Clin Physiol Pharmacol 29:281-290.

Sun PP, Perianayagam MC, and Jaber BL (2009) Endotoxin-binding affinity of sevelamer: a potential novel anti-inflammatory mechanism. Kidney Int Suppl 114: S20-S25.

Swearingen RA, Zhorov E, Cohen A, Sybertz T, and Barry EF (2004) Determination of the binding parameter constants of Renagel capsules and tablets at $\mathrm{pH} 7 \mathrm{by}$ high performance capillary electrophoresis. J Pharm Biomed Anal 35:753-760.

Takebayashi K, Aso Y, and Inukai T (2010) Role of bile acid sequestrants in the treatment of type 2 diabetes. World J Diabetes 1:146-152.

Thomas B (2019) The global burden of diabetic kidney disease: time trends and gender gaps. Curr Diab Rep 19:18.

USRDS (2015) United States Renal Data System Annual Data Report: Epidemiology of Kidney Disease in the United States, National Institute of Diabetes and Digestive and Kidney Diseases, Bethesda, MD.

van Dijk CGM, Oosterhuis NR, Xu YJ, Brandt M, Paulus WJ, van Heerebeek L, Duncker DJ, Verhaar MC, Fontoura D, Lourenç AP, et al. (2016) Distinct endothelial cell responses in the heart and kidney microvasculature characterize the progression of heart failure with preserved ejection fraction in the obese ZSF1 rat with cardiorenal metabolic syndrome. Circ Heart Fail 9:e002760.

Vervloet MG, Sezer S, Massy ZA, Johansson L, Cozzolino M, and Fouque D; ERAEDTA Working Group on Chronic Kidney Disease-Mineral and Bone Disorders and the European Renal Nutrition Working Group (2017) The role of phosphate in kidney disease. Nat Rev Nephrol 13:27-38

Vlassara H, Uribarri J, Cai W, Goodman S, Pyzik R, Post J, Grosjean F, Woodward M, and Striker GE (2012) Effects of sevelamer on HbA1c, inflammation, and advanced glycation end products in diabetic kidney disease. Clin J Am Soc Nephrol 7: 934-942.

Webb NJ, Shahinfar S, Wells TG, Massaad R, Gleim GW, Santoro EP, Sisk CM, and Lam C (2012) Losartan and enalapril are comparable in reducing proteinuria in children. Kidney Int 82:819-826.

Xiao WY, Ning N, Tan MH, Jiang XS, Zhou L, Liu L, Yi D, and Wei P (2016) Effects of antihypertensive drugs losartan and levamlodipine besylate on insulin resistance in patients with essential hypertension combined with isolated impaired fasting glucose. Hypertens Res 39:321-326.

Yilmaz MI, Sonmez A, Saglam M, Yaman H, Kilic S, Eyileten T, Caglar K, Oguz Y, Vural A, Yenicesu M, et al. (2012) Comparison of calcium acetate and sevelamer on vascular function and fibroblast growth factor 23 in CKD patients: a randomized clinical trial. Am J Kidney Dis 59:177-185.

Address correspondence to: Thomas Hübschle, Sanofi-Aventis Deutschland GmbH, Industriepark Höchst, 65926 Frankfurt, Germany. E-mail: Thomas.Huebschle@sanofi.com 